\title{
Reactivity on and of Graphene Layers: Scanning Probe Microscopy Reveals
}

\author{
Oleksandr Ivasenko and Steven de Feyter
}

\begin{abstract}
In this chapter we give an overview of different chemical transformations that can be done on graphene layers and characterized using scanning tunneling (STM) and atomic force microscopies (AFM). We place particular emphasis on the diversity of reactions, systems and synthetic strategies that are now available to surface scientists working in various fundamental and applied research fields. Using imine formation as the model reaction we discuss common principles of building block design and reaction outcomes specific to interfacial synthesis. Then other reactions are briefly overviewed, including: photo- and electrochemically assisted processes, transformations initiated by STM, and finally, reactions involving the covalent modification of graphene layers.
\end{abstract}

\section{Introduction}

Chemistry of and on surfaces is of tremendous importance in material and bio sciences. It will not come as a surprise that surface chemistry takes a special place in nanoscience and nanotechnology as scaling down increases the surface to volume ratio. Among other techniques, scanning probe microscopy tools made it possible to probe surfaces, sometimes at the atomic scale. As these techniques can be used under ambient conditions or even in liquids, they became attractive to chemists and material scientists too. This review aims at giving an overview of molecule-based surface reactivity under ambient conditions or at the liquid-solid interface, on graphite and graphene. While it's not the ambition to give a comprehensive overview, a range of different surface reactivity concepts are highlighted.

O. Ivasenko $(\varangle) \cdot$ S. de Feyter

Department of Chemistry, KU Leuven - University of Leuven,

Celestijnenlaan 200F, 3001 Leuven, Belgium

e-mail: oleksandr.ivasenko@kuleuven.be

S. De Feyter

e-mail: steven.defeyter@kuleuven.be

(C) The Author(s) 2018

D. G. de Oteyza and C. Rogero (eds.), On-Surface Synthesis II,

Advances in Atom and Single Molecule Machines,

https://doi.org/10.1007/978-3-319-75810-7_3 
Below we outline some of the methodological aspects relevant to the topic of this chapter and considered when studying chemical reactivity at interfaces.

\section{Substrates}

Highly oriented pyrolytic graphite (HOPG) is a widely used substrate in scanning tunneling microscopy studies. Conductive, chemically inert, with large (up to hundreds of microns in size) high-quality atomically flat terraces, HOPG is perfectly suited for STM studies and represents an ideal platform for the investigation of various phenomena involving the adsorption on the basal plane (graphene sheets) of graphite. Supramolecular and covalent chemistry developed on HOPG substrates can be easily transferred onto other related materials: various types of graphene, films of reduced graphene oxide, different carbon-based adsorbents (e.g. porous graphitic carbon-PGC), rendering such studies as highly important for the development of future sensors [1, 2], electronic devices [3], catalysts [4], etc.

\section{Molecular design}

Successful STM imaging requires the localization of molecular species on the conductive substrate, preferably in the form of monolayers or ultrathin films. It is quite common to use starting materials that spontaneously form stable monolayers on surfaces. This is achieved using building blocks with extended aromatic cores (for $\pi-\pi$ interactions with the substrates), long alkyl chains (van der Waals intermolecular interactions) or self-complementary hydrogen bonding moieties. In such cases the reaction can be detected with STM via the difference in the monolayer structures of starting materials and products (due to different molecular geometries and intermolecular interactions, see example in Fig. 1).

For the formation of highly ordered films of products strong self-assembly of starting materials could be a curse since monolayer reconstruction entails overcoming additional barriers and often results in multiple monolayer defects. A rather interesting approach is orthogonal building block design in which self-assembly function and reactivity are sufficiently independent. For example, Zimmt et al. have
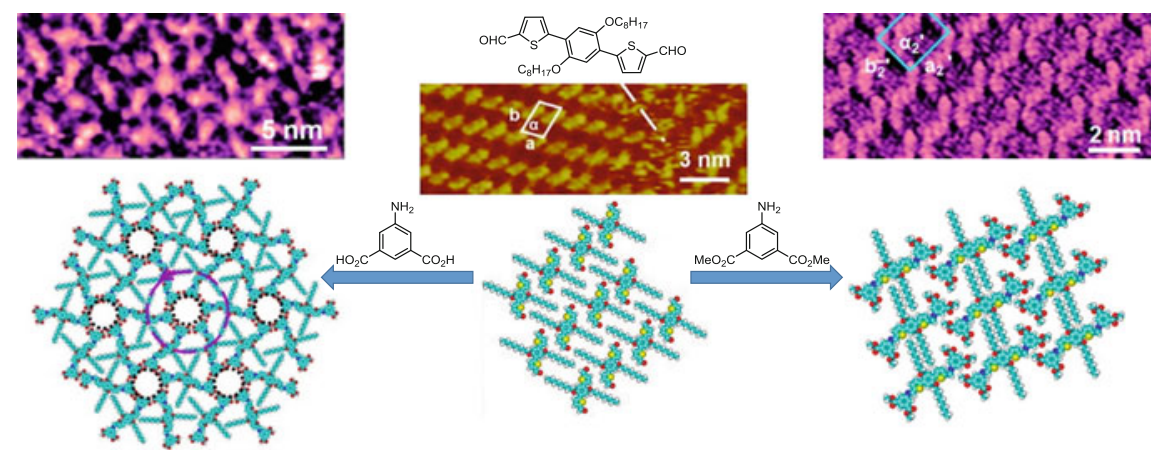

Fig. 1 Distinct monolayer structures of starting dialdehyde and two bis-imine products. Adapted with permission from [5]. Copyright (C) 2013 American Chemical Society 


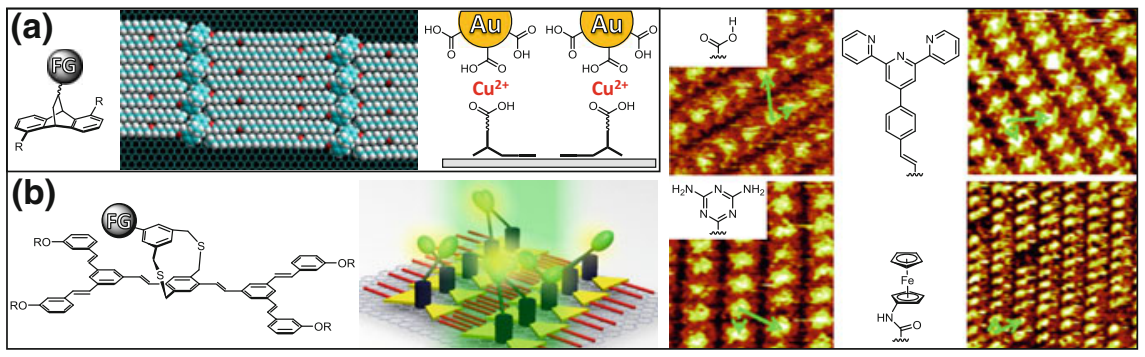

Fig. 2 Examples of building block designs in which self-assembly and reactive functionalities do not interfere with each other. a adapted from [6], copyright (C) 2012, with permission from Elsevier. b adapted with permission from [7, 10]. For [10] copyright (C) 2016 American Chemical Society, for [7] copyright (C) 2014 Wiley-VCH Verlag GmbH \& Co. KGaA

designed carboxy-functionalized building blocks suitable for chemical binding with gold nanoparticles (Fig. 2a, [6]), while the group of Attias has developed a series of original molecular probes bearing optically, electrochemically, hydrogen-bonding and metal-coordination active units (Fig. 2b, [7-10]).

\section{Activation methods}

Similarly to solution-based syntheses, the chemical reactivity of reagents at interfaces can be activated thermally, catalytically (e.g. tuning $\mathrm{pH}$ in acid-catalyzed reaction), photo- or electrochemically. The exact activation method depends on the reaction and often defines the preferred synthetic setup: type of solvent, temperature, work-up, etc. For example, aqueous electrolytes are typically used in electrochemical (EC-STM) experiments, while on-surface syntheses of boroxine and imine polymers can involve solvent-free conditions and delivery of reagents (e.g. water [11], aldehyde [12]) in the form of vapors from the gas phase.

Unique to SPM studies is the use of SPM tips for localized mechanochemical and/or electrical activation of reactants. Such activation is often fully compatible with SPM measurements, and allows nanoscale manipulation and testing of surface structures (Sects. 5, 6 and 7).

\section{Characterization}

Scanning tunneling microscopy is a powerful technique for nanoscale structural characterization of conductive surfaces and monolayers adsorbed on them. In studying chemical reactions, STM can provide accurate (typical uncertainties $\pm 0.1 \mathrm{~nm}$ ) values for unit cells and, in the case of polymers, the length of repeat units. Together with complementary data from DFT modelling or known X-ray structure, the STM measurements can serve as a support of the intended chemical transformation. Adsorbate morphology can be obtained from atomic force microscopy (AFM) or scanning electron microscopy (SEM, e.g. 2.1 Fig. 9) investigation of dry films. 

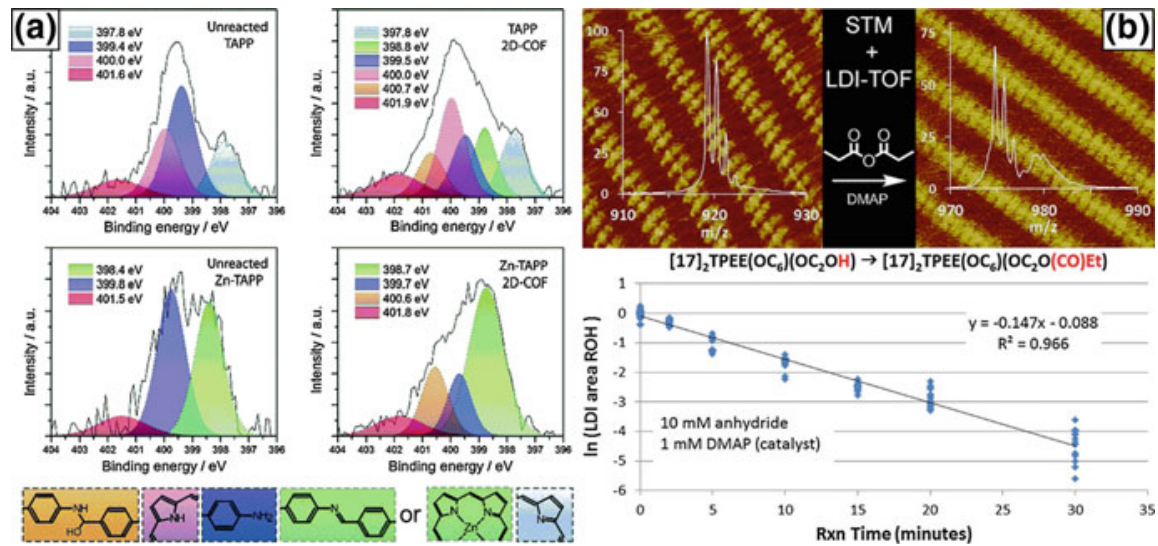

Fig. 3 Examples of STM-complementary characterizations using: a XPS in the synthesis of imine-based 2D COFs [18] — published by The Royal Society of Chemistry. b LDI-TOF in the chemical transformation of a carboxylic building block. Adapted with permission from [19]. Copyright (C) 2017 American Chemical Society

Unfortunately, except for special cases of STM markers [13] - functional groups that have high specific STM contrast and can be used to characterize mixtures of structurally related 'marked' and 'unmarked' molecules [14, 15], STM cannot provide detailed information about the chemical nature of the imaged adsorbates. That is why it is desirable and often mandatory to perform complementary characterization. This could be classical solution techniques, e.g. NMR, provided that there is sufficient amount of material for the analysis [16]. Otherwise, dedicated surface techniques are particularly convenient. Thus, X-ray photoelectron spectroscopy (XPS) has been used to follow formation of polyboroxines [17] and polyimines (Fig. 3a, [18]). Another interesting example is the application of laser desorption/ionization time-of-flight mass-spectrometry (LDI-TOF) and its matrix-assisted modification (MALDI-TOF) for successful characterization of chemical changes in physisorbed monolayers (Fig. 3b, [19]).

\section{Chemical Reactivity}

Since the formation of imines is by far the most studied organic reaction on HOPG, we discuss it in details (Sect. 2.1) covering general aspects pertinent to many other interfacial reactions, and only then provide an overview of other reactions that have been tested (Sect. 2.2). 


\subsection{Synthesis of Imines}

\section{Geometry of the building blocks}

Basic concepts of supramolecular design that relies on the reversible formation of non-covalent interactions are also applicable to the design of new covalent architectures especially when the synthesis occurs with the reversible formation of new covalent bonds and the designed architecture represents the most stable state (thermodynamic control). Formation of imines can be reversible in a number of different regimes: (1) in aqueous solutions with carefully controlled optimal $\mathrm{pH}$ range, (2) at high temperatures in solid state in the presence of water vapors (often using $\mathrm{CuSO}_{4} \cdot 5 \mathrm{H}_{2} \mathrm{O}$ as a source of water), (3) in certain organic solvents at room or slightly elevated temperatures. Thus, a variety of $1 \mathrm{D}$ polymers and $2 \mathrm{D}$ covalent organic frameworks (2D COFs) have been formed from suitably functionalized di-, tri- and tetra-substituted building blocks (e.g. Figure 4). If one of the building blocks is a monoaldehyde or monoamine then the product of condensation is a small molecule (Fig. 1, [5, 20]).

Single $\mathrm{C}-\mathrm{C}$ bonds around imine links are free to rotate, during synthesis such rotations lead to connectivity mismatches and network deformations (defects).

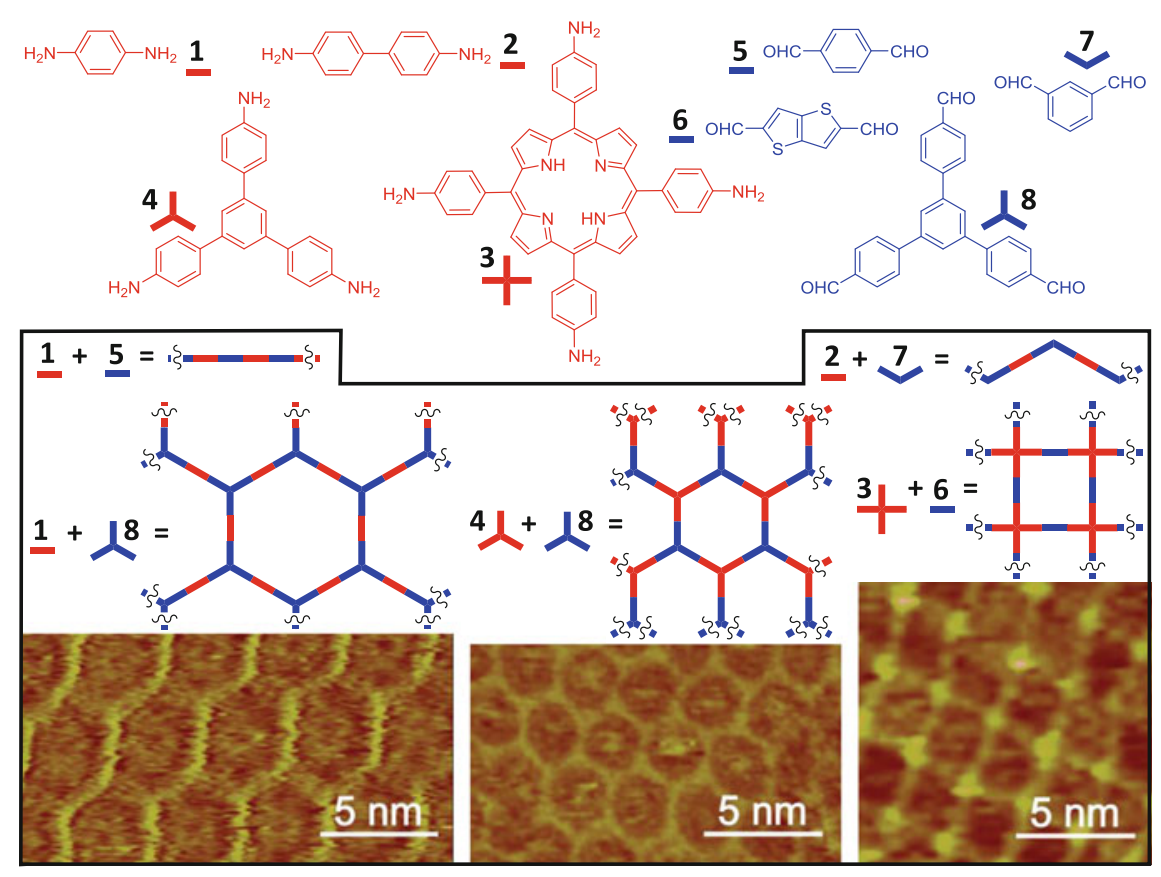

Fig. 4 Examples of the topological design of various imine-based polymeric structures. Adapted from [21], with the permission of AIP Publishing 


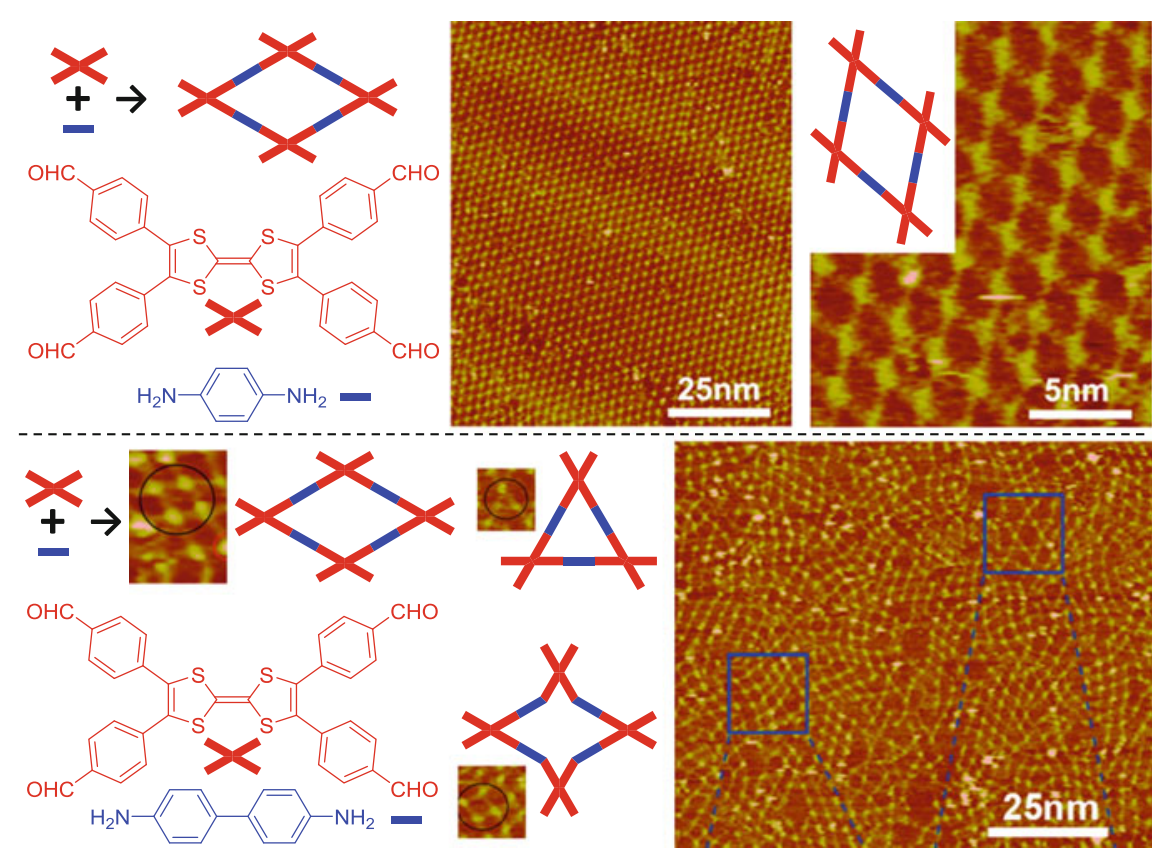

Fig. 5 Longer biphenylene linker results in a larger number of different defects in 2D COF compared to shorter p-phenylene, which forms highly crystalline COF uniform rhomboidal pores. Adapted with permission from [22]. Copyright (C) 2015 American Chemical Society

While every imine COF has defects, their surface density is a function of synthetic protocol (e.g. thermal annealing in the presence of water vapors often allows to overcome energy barriers and improve the quality of polymeric structures [12]) and rigidity of the building blocks (see Fig. 5 and other examples in [22-24]).

Above we have seen how small variations in molecular geometry (rotational disorder along $\mathrm{C}-\mathrm{C}$ bonds) results in significant changes to the structure of reaction products. Larger changes to the building block structure may have drastic outcomes. Thus, very recently, Wang et al. demonstrated that non-planar triamine reacts with dialdehydes at gas-solid interface forming exclusively 1:3 adducts (Fig. 6a, [25]). Varying the concentration, ratio and temperature (up to $200{ }^{\circ} \mathrm{C}$ ) yielded the same adducts, without formation of any other oligomeric or polymeric products. The authors proposed that the rigid tripod geometry restrains the accessibility of terminal groups for further reactions.

\section{Stoichiometry of reagents}

Stoichiometry of reagents in solution can affect various interfacial processes. An interesting example is a kinetically controlled formation of 2:1 diamine:dialdehyde non-covalent co-assembly (Fig. 6b) at room temperature at liquid-solid interface [26] . Such transient structure (life period $<2 \mathrm{~h}$ ) formed only if the diamine:dialdehyde ratio 

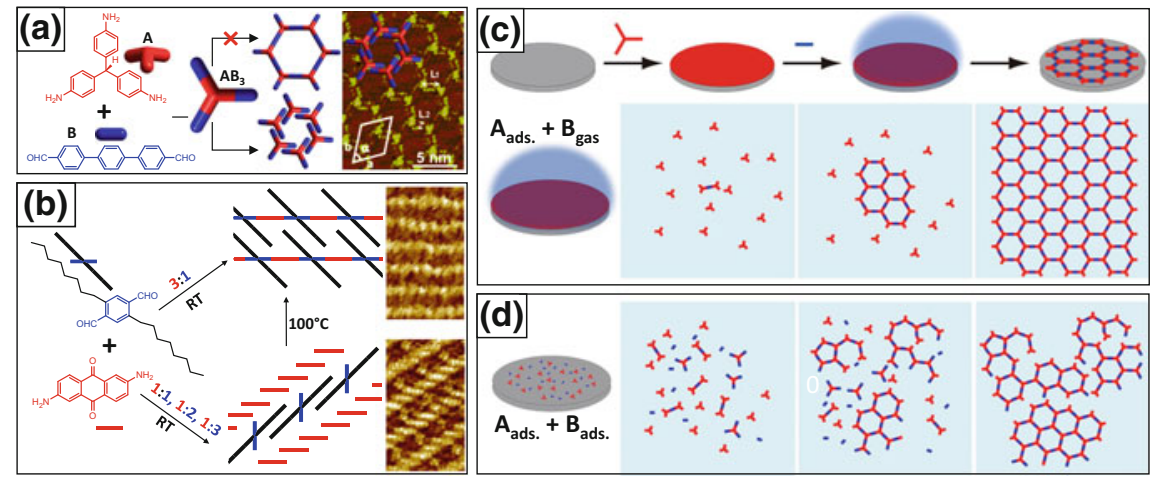

Fig. 6 Examples of: a a thermodynamically stable self-assembly of $\mathrm{AB}_{3}$ adduct. Adapted with permission from [25]. Copyright (C) 2017 Royal Society of Chemistry. b metastable non-covalent co-assembly of a diamine and a dialdehyde. Adapted with permission from [26]. Copyright (C) 2017 American Chemical Society. c The controlled nucleation of 2D COF via a gradual introduction of one of the coupling components versus $\mathbf{d}$ multiple nucleation sites generated upon heating stoichiometric mixtures of both components. Adapted with permission from [12]. Copyright (C) 2013 American Chemical Society

in solution was equal or lower than 1:1. At 3:1 stoichiometry in solution only $1 \mathrm{D}$ polymeric structures were observed on the surface.

Stoichiometry of reagents is also an important parameter in any bi-component reaction. Any large deviation from the ideal stoichiometry should have a negative impact on the yield and degree of polymerization-ultimately resulting in the formation of small functional molecules (e.g. in the reaction of a triamine $\mathrm{A}$ and dialdehyde $\mathrm{B}$ large excess of $\mathrm{B}$ should yield new trialdehyde $\mathrm{AB}_{3}$ - analogous to the one in Fig. 6a, while large excess of A should furnish new tetraamine $A_{2} B$ ). Indeed such structural variations and strong dependency of the product composition on the stoichiometry of reagents were observed in surface-supported imine synthesis on $\mathrm{Au}$ (111) in ultra-high vacuum (UHV) [27]. In majority of the reported imine polymerizations on HOPG however, so strong influence of reagents stoichiometry was not detected. To understand this difference in behavior we should consider the following factors:

- stronger than on HOPG substrate-molecule interactions on Au(111) result in lower on-surface mobility of adsorbed precursors and small oligomers;

- UHV precludes normal pathway (i.e. hydrolysis by dissolved or vaporized water) for reversible formation/braking of imine bonds;

- in the case of reaction at liquid-solid interface there is also additional possibility for preferential adsorption of larger oligomers.

All of them render the abovementioned imine formation in UHV essentially irreversible and strongly dependent on the initial state (stoichiometry, on-surface distribution of precursors); while typical reactions on HOPG are done under reversible conditions, when polymerization is energetically favorable and once 
reaction began it is thermodynamically driven towards large oligomers and polymers.

Stoichiometry of reagents can be intentionally varied during synthesis. Thus, Wan et al., have shown that gradual evaporation of the second component into the excess of the first component already adsorbed on HOPG results in much higher quality COFs than those obtained from direct heating HOPG with mixture of both components [12]. Here, the slow introduction of the second component limits the number of COF nuclei on the surface (Fig. 3c, d) yielding larger domains with fewer interdomain borders and internal defects.

\section{Side-functionality of the building blocks}

Introduction of functional side-groups can result in major alternation of precursor reactivity or properties of the final product. Thus, introduction of phenolic $\mathrm{OH}$ group into imine polymers has been shown (depending on the structure of the building blocks and substitution pattern) to lead to unusual aggregation behavior [28], improved chemical stability (Fig. 7a, [29]) or even alter the chemical nature of the COF through additional chemical transformations (Fig. 7b, [30]).

\section{Dynamic combinatorial chemistry}

In the pioneering work by Samori et al. competitive reactivity of aliphatic diamines with a substituted benzaldehyde in the presence of HOPG was investigated [31]. The authors have shown that thermodynamically less stable self-assembly of bis-imine formed from a short diamine upon addition of longer diamine into the liquid phase undergoes quick trans-imination yielding a more stable self-assembly of bis-imine formed from the longer diamine (Fig. 8a). This simple concept of directing chemical synthesis via preferential adsorption of one of the possible products is very appealing. Unfortunately, so far other systems have shown much

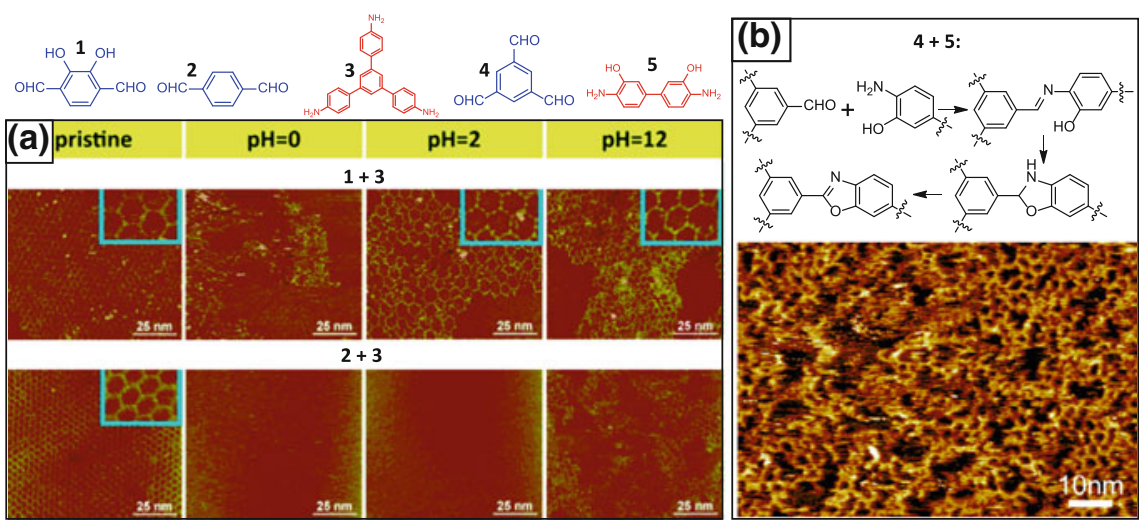

Fig. 7 Improved stability a and post-synthetic transformation $\mathbf{b}$ of $\mathrm{OH}$-functionalized imine 2D COFs. a adapted with permission from [29]. Copyright (C) 2017 Royal Society of Chemistry. b adapted with permission from [30]. Copyright (C) 2015 Royal Society of Chemistry 

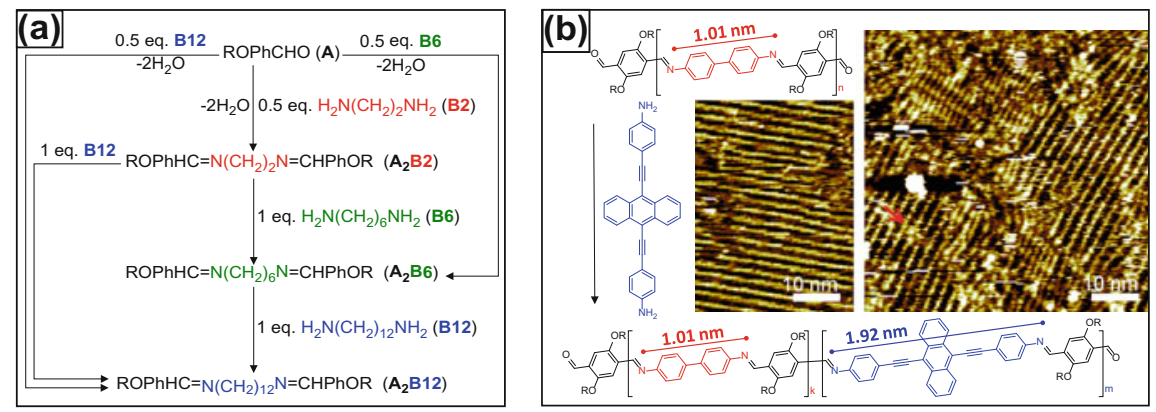

Fig. 8 Examples of dynamic covalent chemistry directed by preferential adsorption. b adapted from [32], copyright (C) 2017, with permission from Elsevier

more complex behavior (Fig. 8b, [32]) when the competition for the surface occurs not only with other reaction products but also with starting material and the selectivity in singling out one of the reaction products is unsatisfactory [16]. Interestingly, in the latter work strong heterogeneous catalysis of imine reactions even for the reaction of weakly adsorbing terephthalic aldehyde and 3,5-bis (tert-butyl)aniline was observed. Currently we investigate if adsorbents ( $\mathrm{PGC}, \mathrm{SiO}_{2}$, etc.) can serve as general catalysts of other reactions besides formation of imines.

\section{Formation of multilayers and thin films}

Non-homogeneous deposition of precursors will result in heterogeneous polymeric films with varied thickness throughout the surface. Lei et al. developed a general and convenient methodology for the optimization of room temperature polymerizations at liquid-solid interface in which, keeping the ratios amine:aldehyde fixed, the total concentration is carefully tuned for optimal polymer quality [33]. Using high total concentrations it was also possible to grow multilayers.

We have already mentioned the formation of highly ordered COF monolayer from substituted tetrathiafulvalene (TTF) tetraaldehyde and p-phenylenediamine (Fig. 5). Surprisingly growth of the second layer appears to be templated by the first and results in eclipsed stacking. Stacking was the result of strong and specific $\pi-\pi$ interactions between TTF cores, as convincingly demonstrated by spontaneous self-assembly of unsubstituted TTF on top of TTF sites of the COF monolayer [36]. The authors have noticed that the quality of the bilayer was higher if the polymerization was done in a stepwise fashion, otherwise monomer stacking and polymerization occur simultaneously leading to much more disordered COF films.

The morphology of the polymeric films also depends on the deposition method itself. Kunitake et al. have demonstrated that, unlike gas phase deposition leading to uniform films, deposition from liquid phase $(\mathrm{pH}$-controlled synthesis in aqueous solutions) yields sponge-like structures (Fig. 9a, [34, 37]).

Finally, it should be mentioned that micropatterns of polymeric films can be deposited on any arbitrary substrate using lithographically controlled wetting or conventional ink-jet printing (Fig. 9b, [35]). 


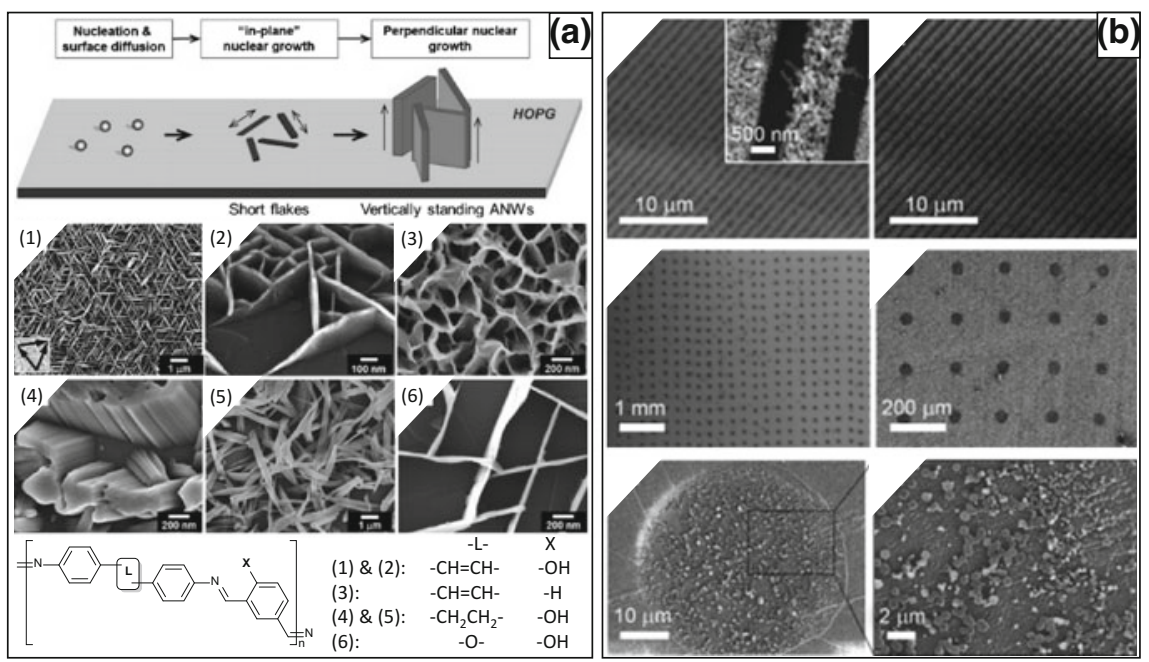

Fig. 9 Examples of $\mathbf{a}$ different growth and $\mathbf{b}$ lithographic deposition of polymeric films. a adapted from [34], copyright (C) 2013, with permission from Elsevier. b adapted with permission from [35]. Copyright (C) 2015 Wiley-VCH Verlag GmbH \& Co. KGaA

\subsection{Other Reactions}

One of the simplest chemical transformation is protonation. Li et al. have shown that addition of strong acids $\left(\mathrm{HNO}_{3}, \mathrm{CH}_{3} \mathrm{SO}_{3} \mathrm{H}, \mathrm{CF}_{3} \mathrm{SO}_{3} \mathrm{H}\right)$ greatly accelerates the self-assembly of neutral di-pyridyl building blocks with protonated intermediates acting as active species directing on-surface self-assembly [38]. Formation of copper salt linkers was used for nanopatterned assembly of functionalized gold nanoparticles on top of strongly physisorbed monolayers on graphite (Fig. 2a, [6]).

In many aspects metal coordination is similar to protonation. In situ generation of potassium coordination complexes at HOPG-solution interface was demonstrated for valinomycin (Fig. 10a, [39]), G 4 quartets of 9-(n-octadecyl)guanine (Fig. 10b, [40]) and tetraethylene glycol derivative (mimicking the complexation of crown ethers, Fig. 10c, [41]).

Various nitrogen-containing building blocks have also been explored to create surface nanopatterns of ' $0 \mathrm{D}$ ' metal complexes [45], to template the nucleation of metallic nanoparticles (Fig. 10d, [42]), to synthesize 1D coordination polymers (Fig. 10e, [43]) and 2D metal-organic frameworks (MOFs, Fig. 10f, [44]).

Procedure-wise one of the simplest organic transformations are thermally induced intramolecular isomerizations. Heating is all that is necessary to consider. An interesting example of such isomerizations is tandem Claisen-Hiratani rearrangement - a [3]-sigmatropic rearrangement in which an allyl aryl ether is converted thermally to allylphenoles forming new $\mathrm{C}-\mathrm{C}$ bond [46-48]. 

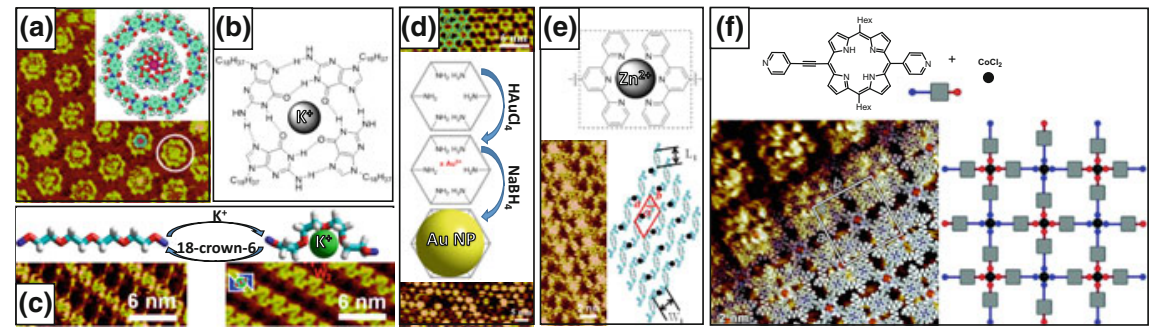

Fig. 10 Formation of coordination complexes at HOPG-solution interface. a Potassium-valinomycin as guest inside a macrocycle. Adapted from [39] with permission of The Royal Society of Chemistry. Copyright (C) 2013 Royal Society of Chemistry. b dynamic assembly-reassembly of substituted G4 quartets [40]. c Nanoscale actuation of oligoether 'springs'. Adapted from [41] with permission of The Royal Society of Chemistry. Copyright (C) 2014 Royal Society of Chemistry. d Pre-concentration of $\mathrm{Au}^{3+}$ with subsequent templated reduction to Au nanoparticles. Adapted from [42] with permission of The Royal Society of Chemistry. Copyright (C) 2015 Royal Society of Chemistry. e On-surface synthesis/self-assembly of 1D (adapted with permission from [43]; copyright (C) 2015 American Chemical Society) and 2D (adapted from [44] with permission of The Royal Society of Chemistry; copyright (C) 2014 Royal Society of Chemistry) metalorganic supramolecular polymers

Analogues to imine formation, $\mathrm{C}=\mathrm{C}$ condensation of an aromatic dialdehyde and barbituric acid (Knoevenagel reaction) was investigated at liquid/HOPG and vapor/ HOPG interfaces [49]. Here STM was successfully used for quantitative analysis of on-surface distribution of reaction products.

Also the synthesis of 2D COFs on carbon-based surfaces is not limited to imines only. A very popular reaction for the synthesis of surface-supported COFs is the formation of boroxines from different boronic acids (Fig. 11a, [17, 50, 53]). An interesting development is the use of boroxine acid esters for in situ hydrolysis and polymerization [11]. Thin films via bi-component condensations of boronic acids and hexahydroxytriphenylene (HHTP) were first prepared as early as 2011 [54], but only very recently formation of monolayer films of such 2D-COFs demonstrated to take place under mild conditions at liquid-solid interface (Fig. 11b, [51, 55]). Finally, it is possible to 'marry' different polymerization chemistries: 3 - and 4-formylphenylboronic acids react with a triamine building block forming isomeric imine-boroxine hybrid 2D COFs (Fig. 11c, [52]).

\section{Photochemical Reactivity}

Presence of azobenzene moieties in derivatives is the calling card of a photoswitching functionality anticipated in the design. Indeed, cis-trans isomerization of $\mathrm{N}=\mathrm{N}$ bond in azobenzenes is a well-defined process free from any side-reaction or degradation. For example isophthalic acid derivative were shown to form stable self-assembly that upon irradiation undergo reversible isomerization [56, 57]. The 


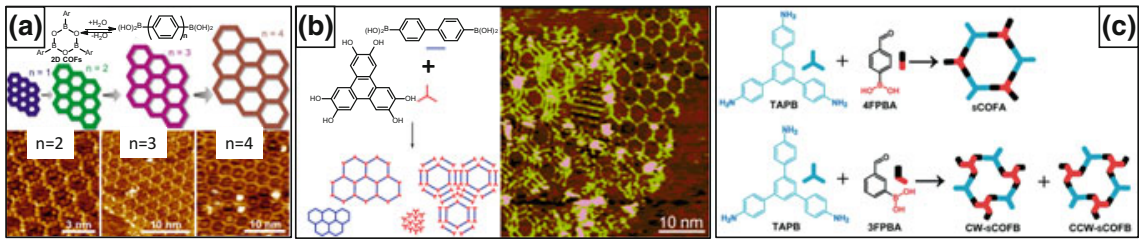

Fig. 11 Boronic acid based 2D COFs on HOPG via a dehydration of boronic acids into boroxines, b polycondensation of diboronic acids and hexahydroxytriphenylene (HHTP), c simultaneous formation of boroxine and imine linkages from the reaction of bifunctional building blocks. a adapted with permission from [50]. Copyright (C) 2012 American Chemical Society. b adapted from [51] with permission of The Royal Society of Chemistry. Copyright (C) 2016 Royal Society of Chemistry. c adapted from [52] with permission of The Royal Society of Chemistry. Copyright (C) 2017 Royal Society of Chemistry

assemblies of corresponding cis- isomers have different structure and lower stability compared to trans-. The latter is due to non-planarity of cis-azobenzene resulting in less efficient substrate molecule interaction. Alternative designs (Fig. 12a) when cis-trans isomerization is occurring on azobenzene units pointing away from the surface into the solution phase were also realized [58, 59].

More complex supramolecular architectures were also tested. Thus, exploring the host-guest concept, assemblies of photoactive host (Fig. 12b, [60, 63]) and guest (Fig. 12c, [61, 64]) were studied. In the former example photoisomerization was demonstrated to influence surface adsorption of a small molecular probe (coronene), while the latter example illustrated variability and responsiveness of the flexible host network to differently shaped isomeric guests. Exploring formation of supramolecular polymers, Yamauchi et al. demonstrated phototriggered supramolecular polymerization of a naphthalene-azobenzene dyad [62]. Here cis-trans photoisomerization allowed to produce shorter polymeric fibers with narrower polydispersity compared to those generated by thermal polymerization (Fig. 12d).

Stilbene moiety, being structurally similar to azobenzene can also undergo cis-trans photo-isomerization. In dilute solutions this is a typical outcome of photoirraditation of various building blocks with olefinic $\mathrm{C}=\mathrm{C}$ bonds. However, in dense assemblies (on surface or in crystals), reactions can go further and produce covalent dimers (e.g. Fig. 13a, [65, 66]). Such transformations are often topochemical. The required close proximity and proper orientation of reacting bonds can be achieved through supramolecular design, e.g.: for efficient on-surface photocoupling supramolecular dimers of 1,2-bis(4-pyridyl)ethylene (BPE) bound by two isophthalic acid molecules were confined in a porous self-assembly on HOPG surface (Fig. 13b, [67]).

Photodimerizations can involve reconstruction of multiple bonds as in the photodimerization of anthracenes (Fig. 13c, [68]) or arylethenes (Fig. 13d, [6971]) and in the metal-free on-surface photochemical homocoupling of 4-ethynylbenzoic acid (Fig. 13e, [72]). 

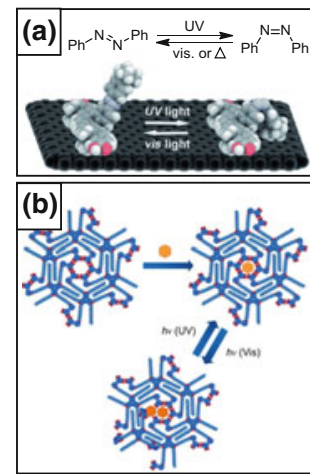

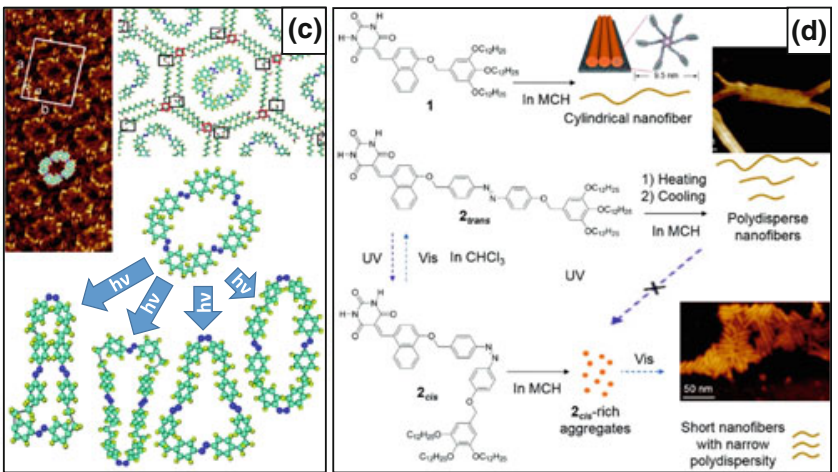

Fig. 12 The application of azobenzene units in a structural phototransformations of physisorbed monolayers, $\mathbf{b}$ the release/adsorption of guests; $\mathbf{c}$ tuning supramolecular network of the host in response to photoisomerization of the guest; d controlling the growth and morphology of nanofibers. a adapted with permission from [59] - published by the PCCP Owner Societies. b adapted with permission from [60]. copyright (C) 2013 Wiley-VCH Verlag GmbH \& Co. KGaA. c adapted with permission from [61]; copyright (C) 2009 American Chemical Society. d adapted with permission from [62]; copyright (C) 2017 The Chemical Society of Japan

Going beyond the formation of small molecules, it is possible to design 1,3-diacetylene building blocks that undergo topochemical photopolymerization when assembled on graphite [56]. Building blocks with one diacetylene unit produce 1D polymeric chains (Fig. 14a), while introduction of two or more reactive units upon exhaustive photopolymerization can furnish non-porous 2D COFs (Fig. 14b, [73]). In the latter example formation of polydiacetylene and polyacetylene chains was assumed. Zimmt et al. demonstrated that 1,3-diacetylene units are also powerful supramolecular synthons (Fig. 14c, [74]), that, together with the possibility of photopolymerization (Fig. 14d, [75]), makes them very interesting for versatile surface nanopatterning.

Combination of azobenzene and diacetylene units within one building block resulted in reach photochemical behavior of on-surface assemblies [77]. Depending on the irradiation wavelength, diacetylene polymerization $(v=254 \mathrm{~nm})$ or reversible trans-cis $(v=365 \mathrm{~nm}) /$ cis-trans $(v=435 \mathrm{~nm})$ isomerizations of azobenzene cores can be triggered (Fig. 14e).

\section{Electrochemical Reactivity}

Good chemical stability and electrical conductivity of graphene sheets makes carbon-based electrodes (HOPG, graphitic rods, glassy-carbon, graphene on different supports, etc.) interesting for various electrochemical (EC) transformations.

Both inorganic [78] and organic nanostructures [79] on surfaces can be directly prepared by electrochemical synthesis. Supramolecular monolayers bearing 

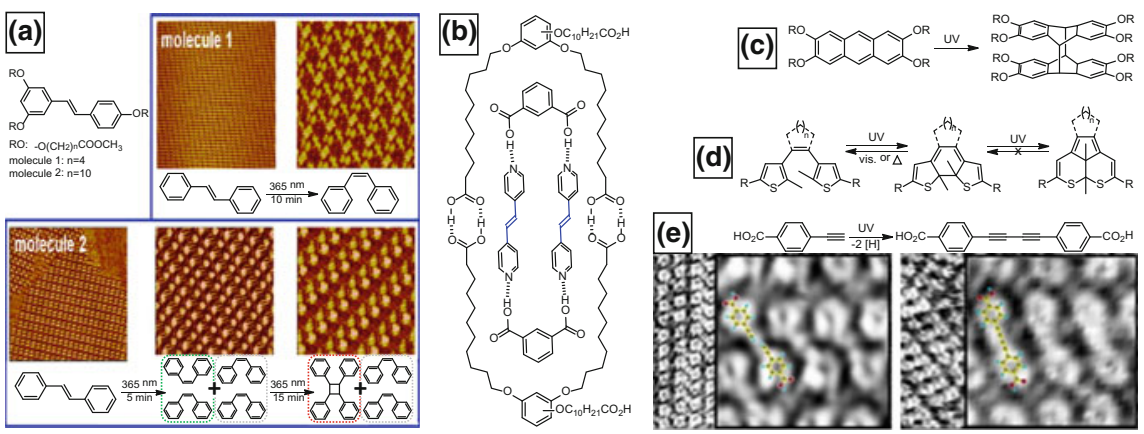

Fig. 13 Photochemical transformation of covalent bonds: a supramolecular network in which stilbene units undergo isomerization and partial dimerization; b oriented and confined inside supramolecular host pores BPE molecules readily dimerize upon photoirradiation [67]; c, d the photochemistry of anthracenes and arylethenes that has already been explored at HOPG-solution interface in [68] and [69-71], respectively; e photoinitiated metal-free oxidative coupling of acetylenes. a adapted with permission from [65]; copyright (C) 2014 American Chemical Society. e adapted with permission from [72]; copyright (C) 2016 American Chemical Society
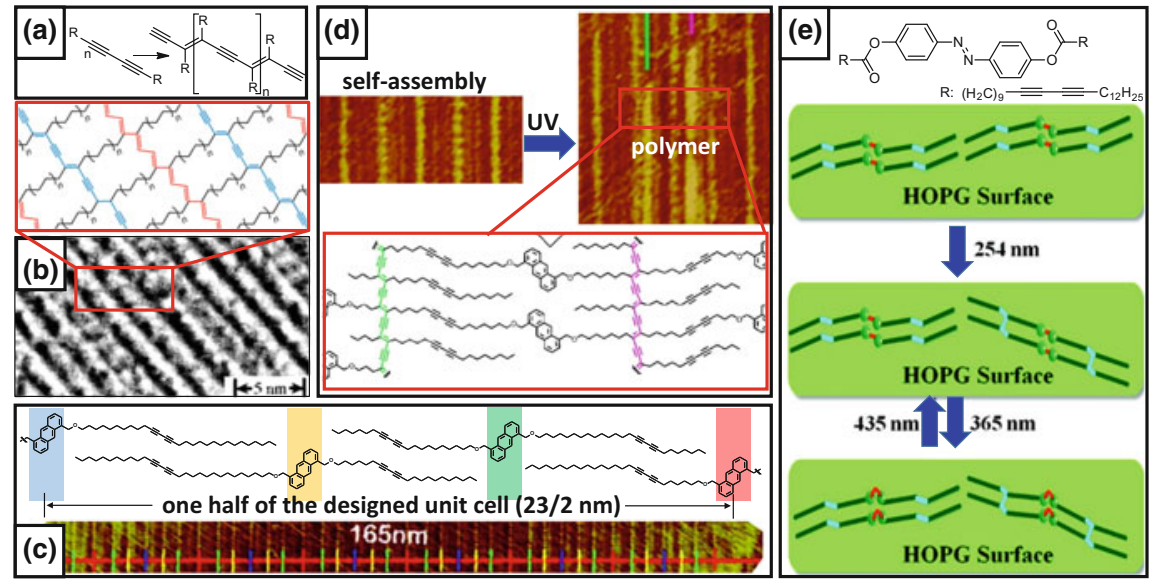

Fig. 14 Photopolymerization of diacetylene assemblies: a general reaction scheme; $\mathbf{b}$ model and STM of a 2D covalent frameworks; c design and $\mathbf{d}$ photopolymerization of four-component co-assembly; e building block with dual photofunctionality bearing both diacetylene and azobenzene units. b, c, d, e were adapted with permission from [73-STM image, 76-model], [74], [75] and [77], respectively. [73] copyright (C) Wiley-VCH Verlag GmbH \& Co. KGaA. [74] copyright (C) 2012 American Chemical Society. [75] copyright (C) 2015 American Chemical Society. The model in (b) adapted by permission from Macmillan Publishers Ltd: Nature Chemistry [76], copyright (C) 2013. [77] copyright (C) 2012 American Chemical Society 
electrochemically active groups (Fig. 15a, [80]) can be prepared by conventional self-assembly from solution and characterized using STM (structure) and cyclic voltammetry ( $\mathrm{CV}$, electrochemical properties).

The electrochemical STM (EC-STM, Fig. 15b) on atomically flat substrates (HOPG, graphene) is particularly suitable for structural characterization of interfacial redox processes. For example, recently we have reported in situ generation and nanoscale characterization of dicationic, monocationic and neutral assemblies of dibenzylviologen (Fig. 15c, [81]). Another recent example is the EC-STM surface characterization in a study of electrocatalytic activity of porphyrins adsorbed on graphene/Au(111) (Fig. 15d, [82]).

\section{Tip-Induced Reactivity}

Besides powerful imaging, scanning probe microscopies are also capable of nanoscale modification of on-surface structures. In STM, the tip can locally modify the matter because of microscopic dimensions (mechanical actuation, also possible for AFM), large $10^{7}-10^{8} \mathrm{~V} / \mathrm{cm}^{2}$ electric field (electrostatic polarization), high current densities (electronic excitation), local heating or any combination of mentioned pathways. Often the exact origin of tip-induced changes is hard to determine and can only be guessed from the chemical nature of on-surface structures and type of nanoscale modifications.

Changes can be limited to non-covalent interactions in physisorbed assemblies, e.g.: (a) movement of single $\mathrm{C}_{60}$ molecules between the pores of a host network (Fig. 16a, [83]); (b) shattering a large domain of a supramolecular assembly into many smaller ones (Fig. 16b, [15]); (c) using electrical pulse to trigger formation of

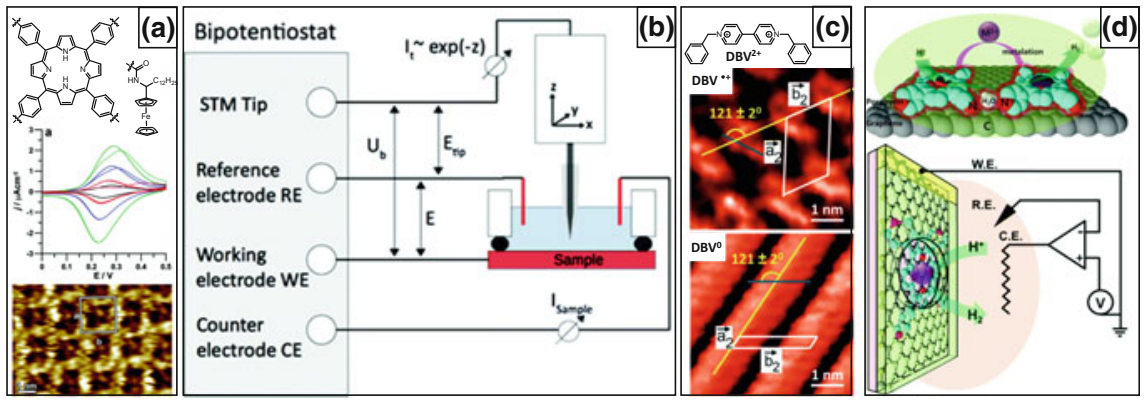

Fig. 15 a CV and STM of self-assembled porphyrin functionalized with electrochemically-active ferrocene moieties [80]. EC-STM operational scheme (b, [81]) and application for in situ generation of reduced viologens (c, [81]) and probing the catalytic activity of porphyrins on graphene (d, [82]). [80] — published by The Royal Society of Chemistry. [81] — published by The Royal Society of Chemistry. c adapted from [82] with permission of The Royal Society of Chemistry. Copyright (C) 2017 Royal Society of Chemistry 
a metastable polymorph (Fig. 16c, [84]); (d) lithographic patterning of a supramolecular host-guest multilayer (Fig. 16d, [85]).

Chemical reactions on HOPG surface are also possible. STM imaging at $-800 \mathrm{mV}$ was sufficient for sporadic reduction of $\mathrm{Mn}$ (III) porphyrins to $\mathrm{Mn}$ (II) states and characterization of different oxygenated species thereof (Fig. 17a, [86]). An example of much more precise tip-induced reaction is [2+2]-oligomerization of $\mathrm{C}_{60}$ by electric pulses through its tri-and tetralayers. Nakaya et al. demonstrated unprecedented control over writing (polymerization) and erasing (depolymerization) of nanoscale structures (Fig. 17b, [87]), promoting such systems for $>$ Tbits/in ${ }^{2}$ aerial density non-volatile storages that can potentially be scaled to writing and erasing speed above Mbits/s or even Gbits/s.

1D polymerizations can be initiated by electrical pulsing over diacetylene self-assembly [88]. The reaction is highly localized allowing initiation of specific diacetylene rows. Monolayer defects interrupt polymerization, while presence of suitable molecules (phthalocyanine [89], $\mathrm{C}_{60}$ [90]) on the way of reactive chain end results in 'molecular soldering': covalent $\mathrm{C}-\mathrm{C}$ coupling of small molecules and polymeric 'wire' (Fig. 18a). Finally, the layers of boroxine 2D COF and its host-guest co-assembly with $\mathrm{C}_{60}$ were tailor-cut using STM lithography (Fig. 18b, c, [91]). The mechanism is not known, presumably the cutting happens via the oxidation of C-B bonds.
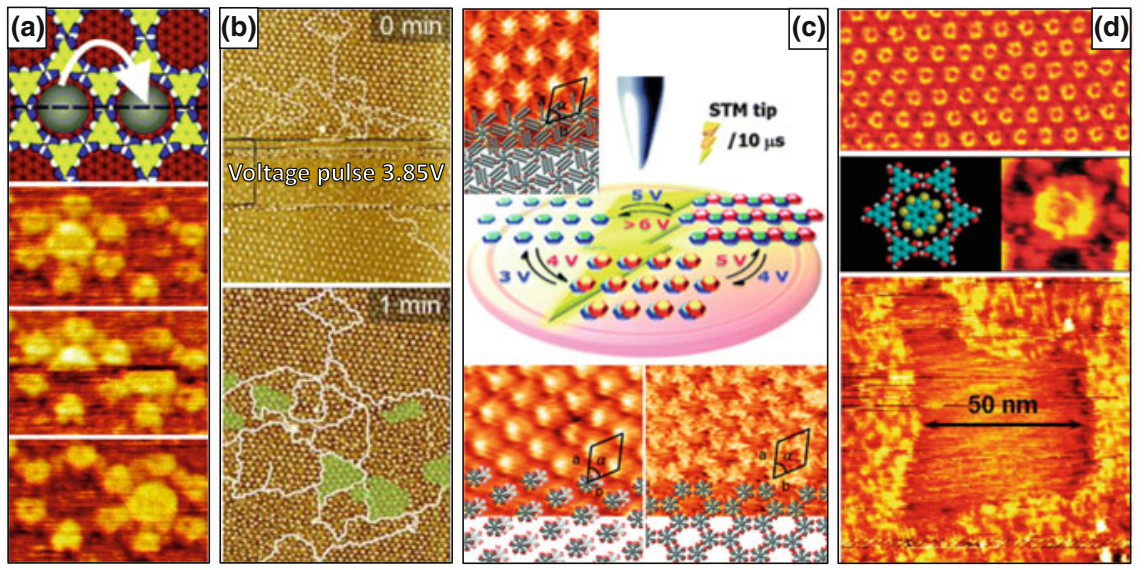

Fig. 16 STM manipulations that do not involve making/braking of covalent bonds: a physical displacement of single molecules [83]; pulse-induced desorption-readsorption of many molecules that leads to the formation of the same (b, [15]) or polymorphic (c, [84]) assemblies; d local removal of a supramolecular multilayered films [85]. a adapted with permission from [83]; copyright (C) 2017 The Chemical Society of Japan. b adapted with permission from [15]. Copyright (C) 2016 Nature Publishing Group. c adapted from [84] with permission of The Royal Society of Chemistry. d adapted from [85] with permission of The Royal Society of Chemistry 


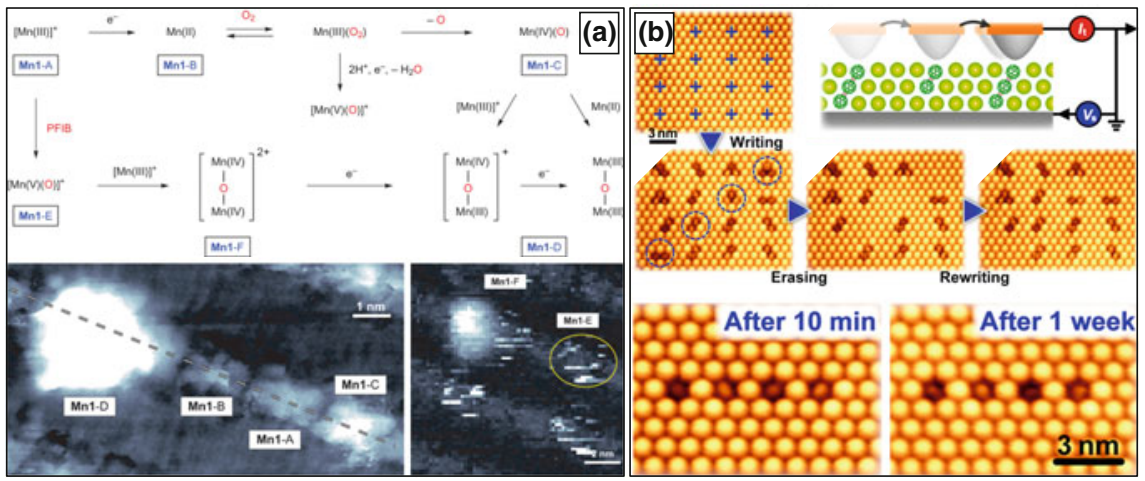

Fig. 17 STM in a the investigation of redox chemistry of $\operatorname{Mn}(111)$ porphyrin [86]; and $\mathbf{b}$ in reliable, spatially localized and precise oligomerization of $\mathrm{C}_{60}$ molecules in multilayered films [87]. a adapted with permission from [86]. Copyright (C) 2013 Nature Publishing Group. b adapted with permission from [87]. Copyright (C) 2016 The Japan Society of Applied Physics
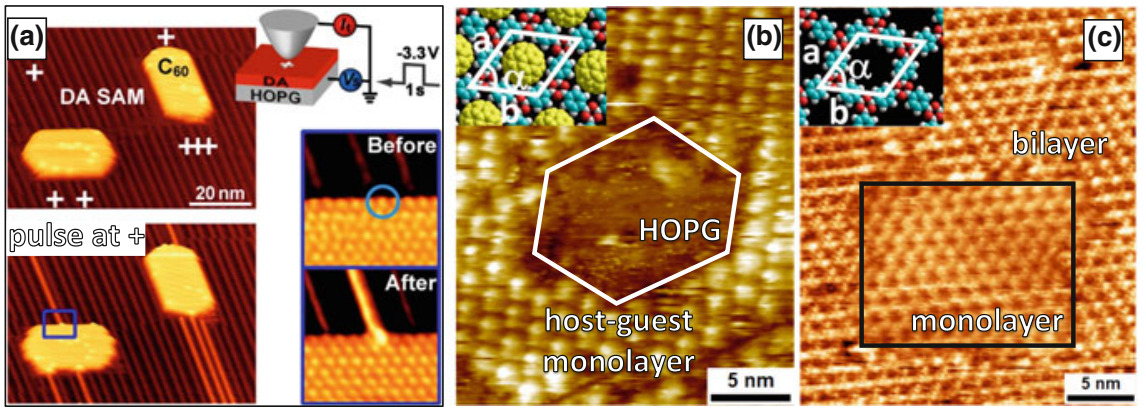

Fig. 18 STM manipulations involving reconstruction of covalent bonds: a pulse-induced polymerization of diacetylene assemblies can be used to covalently 'solder' polydiacetylene 'wires' to physisorbed molecules of $\mathrm{C}_{60}$ [90]; continuous scanning under appropriate tunneling settings was used to locally scratch boroxine 2D COF layers $\mathbf{c}$ and its host-guest assemblies with C $_{60}$ [91]. a adapted with permission from [90]; copyright (C) 2014 American Chemical Society. b, c were adapted from [91] with permission from The Royal Society of Chemistry

\section{Graphene Manipulation and Grafting}

Graphene is not completely inert and under certain conditions can undergo chemical reactions. High temperature $\left(\geq 400{ }^{\circ} \mathrm{C}\right.$, e.g. [92]) or oxygen plasma oxidation [93] of HOPG in air results in random pits on the surface. Much better control over the size and localization of surface pits can be achieved via the spatially patterned ion-bombardment Fig. 19a, [94]). Pits in graphite were used as 2D corrals for studies of molecular self-assembly [95] and even for confined polymerization of diacetylenes (Fig. 19b, [96]). If the oxidation is performed on 
surfaces pre-covered with patterned assemblies of other materials (nanoparticles [97], wires [98], polymers [99], DNA assemblies [100], for more examples please refer to the recent review [101]) the latter act as lithographic masks producing corresponding patterns in graphene layers. Also, STM can be used to rip and fold small parts of graphene sheets (e.g. by $7 \mathrm{~V}$ voltage ramp with sequential scanning, Fig. 19c, [102]) or to lithographically burn-out arbitrary shapes with nm precision (by continuous scanning in air with bias $\geq+2$ V, Fig. 19d, [103, 104]).

Grafting-covalent functionalization of graphene layers via partial conversion of $\mathrm{sp}^{2}$ carbons to $\mathrm{sp}^{3}$ - is very interesting for various applications. Here we will talk exclusively about radical arylation using aryldiazonium precursors. This is because it is one of the most versatile (easy accessibility of starting anilines) and methodologically developed graphene functionalizations. Nevertheless, the discussed design concepts, preparation strategies and SPM characterization should be also applicable to other grafting chemistries (carbenes, nitrenes, Diels-Alder adducts, etc. [105]).

Grafting chemistry is quite simple: aryldiazonium cations through one-electron reduction (usually electrochemical [106], although using reductants is also possible [107]) transform into corresponding aryl-radicals which are sufficiently reactive to attack accessible graphene surface. For characterization it is important to use combination of complementary techniques, e.g.:

- Raman spectroscopy to assess global uniformity of grafting (also for rough estimation of the grafting density);
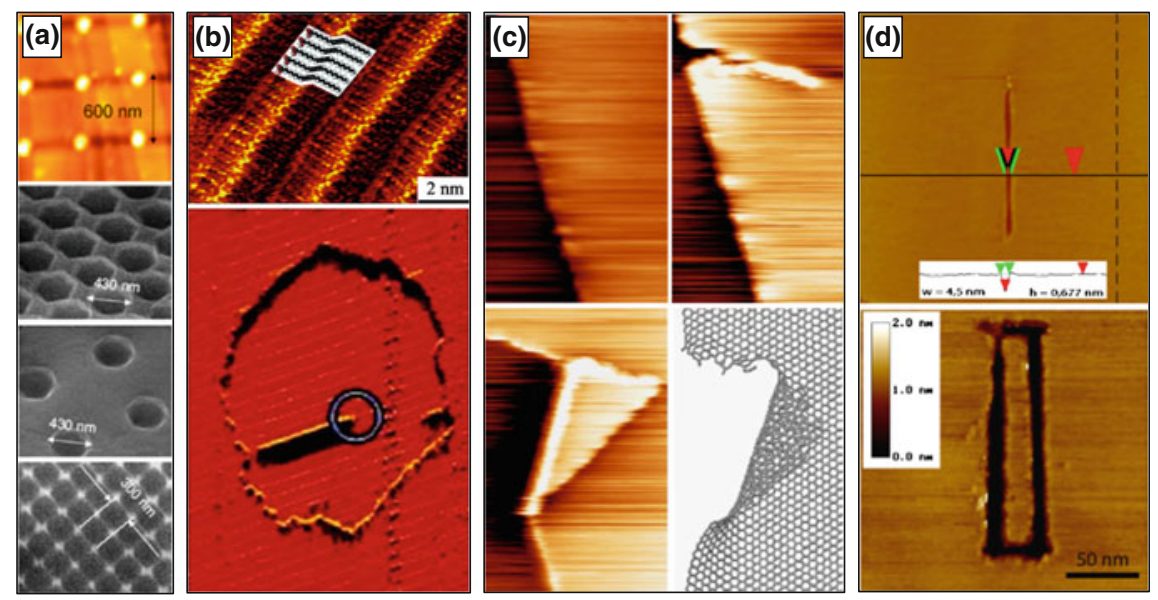

Fig. 19 a Advanced patterned oxidation of graphitic substrates [94]. b The use of corrals in HOPG for the confinement of tip-induced polymerization of diacetylene assemblies [96]. Direct exfoliation (c, [102]) and oxidative etching (d, [103]) of graphene layers with STM tip. a adapted with permission from [94]; copyright (C) 2006 IOP Publishing Ltd. b adapted with permission from [96]; copyright (C) 2005 American Chemical Society. c adapted from [102] with permission from the PCCP Owner Societies, copyright (C) 2017. d adapted with permission from [103]; copyright $(C$ 2017 Wiley-VCH Verlag GmbH \& Co. KGaA 
- $A F M$ to determine film thickness (via mechanical scratching of grafted film), morphology and roughness;

- $S T M$ to prove nanoscale arrangement of grafted species, to distinguish them from physisorbed impurities and for spatially controlled degrafting (see below);

- XPS to prove chemical nature/integrity of functional groups (after grafting or any subsequent treatment of the grafts).

In STM, grafted aryls appear as bright blobs (brighter than physisorbed benzene derivatives $[108,109])$ and covalent attachment to the surface underneath can be seen from characteristic local perturbation of graphene lattice (Fig. 20a). Thickness of the grafted adlayer primarily depends on the aryl structure: aryl radicals with 3-, 4-, 5-positions blocked to radical attack form well-defined non-dendritic grafts [110]. Grafting density can be easily tuned by changing grafting conditions (Fig. 20b), approaching the maximum at $\sim 2-4$ grafts $/ \mathrm{nm}^{2}$. Substituents can be varied widely including among the most unusual examples: directly graftable diazonium salts bearing free-radical moieties (Fig. 20c, [111]), metal complexes (Fig. 20d, [112]) and phosphomolybdate clusters (Fig. 20e, [113]). If necessary, functional groups on grafted aryls can be further modified by adapting surface functionalization protocols developed for organic synthesis on solid supports. Such advanced functionalization has already been successfully used for the design of various sensors [114].

Water soluble diazonium salts can be grafted from aqueous solutions (often using dilute $\mathrm{HCl}$ as supporting electrolyte). Water-insoluble precursors can be electrochemically grafted from organic solvents (e.g. acetonitrile, tetrahydrofuran, etc.) containing quaternary ammonium salts as supporting electrolytes. Very recently Ejigu et al. have shown that using DMSO as solvent and $\mathrm{CsClO}_{4}$ as electrolyte results in simultaneous grafting and exfoliation of graphene layers [115].

Molecular adsorbates can affect grafting in two ways: by preventing the grafting of graphene lattice directly underneath the molecules, and by directing the nanostructured functionalization of self-assembly defects and interdomain borders (Fig. 21a, b, [108]). Here, n-pentacontane was chosen as the molecular adsorbate because of high chemical inertness (typical for all linear alkanes) and its strong adsorption. The latter ensured that reactive species, by- and side-products will not displace the molecular template. Using derivatives capable of ordered self-assembly (Fig. 20d) opens new possibilities for nanostructured surface functionalization during direct grafting [112] or through pre-assembly of diazonium precursor (Fig. 21c, [116]). In the last two examples it is unfortunate that STM was not used to prove the covalent nature of the observed structures and to assess the density/ arrangement of molecules that are actually grafted.

Different nanostructured grafting should be accessible via soft lithography using nanoparticle assemblies as templates. While there is yet no example on graphene-based substrates, the same Ru complex (Fig. 20d) was reduced onto ITO pre-covered with a colloidal self-assembly of polystyrene beads [112]. After the removal of templates patterned thin film with circular nanowells (periodicity $\sim 1 \mu \mathrm{m}$, diameter $\sim 350 \mathrm{~nm}$ ) was successfully prepared (Fig. 22a). 

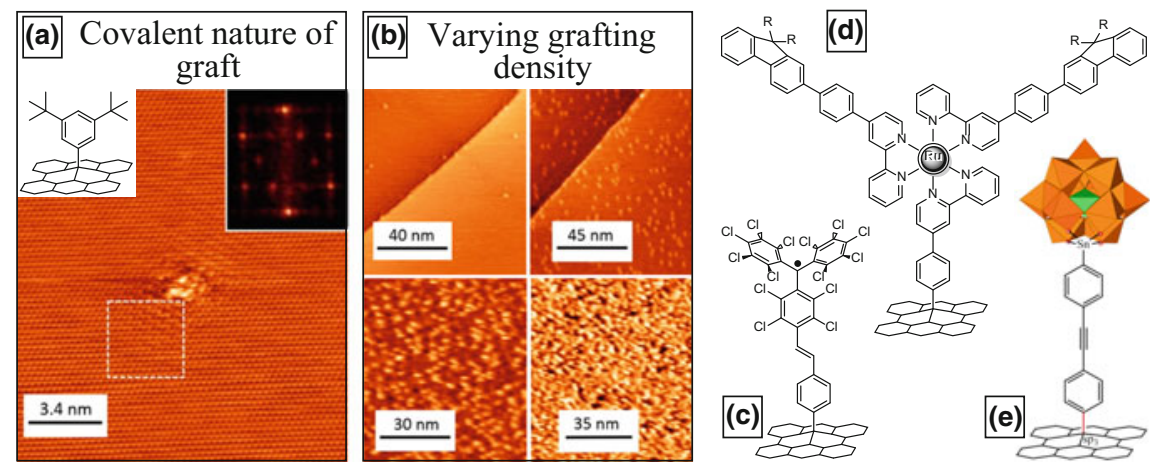

Fig. 20 Functionalization of graphene layers via covalent grafting of aryls: a distortion of graphene lattice in the vicinity of the graft as the evidence of the covalent nature of graft attachment (inset shows 2D FFT of the distorted lattice) [110]; b altering grafting conditions allows controlled tuning of the grafting density [110]. Some of the most unusual grafts feature: $\mathbf{c}$ a stable free radical [111]; d a metalorganic complex [112]; e a phosphomolybdate cluster [113]. a, b adapted with permission from [110]; copyright (C) 2015 American Chemical Society. e adapted with permission from [113]; copyright (C) 2016 American Chemical Society
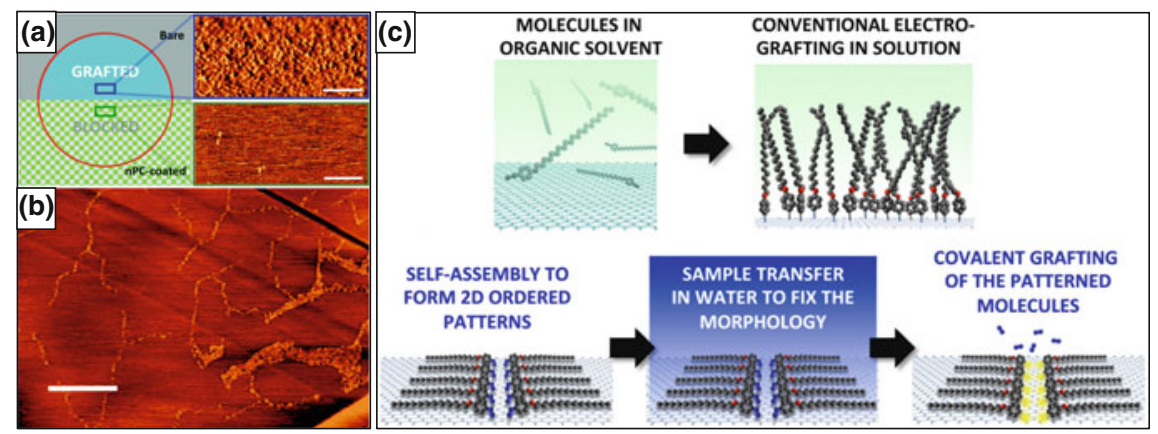

Fig. 21 Molecular self-assembly and covalent grafting: a the protection of graphene layers from the attack of aryl radicals by physisorbed self-assembly of n-pentacontane $\left(\mathrm{nPC}, \mathrm{n}-\mathrm{C}_{50} \mathrm{H}_{102}\right)$ and b nanostructured grafting as the result of covalent grafting of HOPG surface at the defects and interdomain borders of nPC monolayer [108]. Preorganization of diazonium salts via on surface self-assembly leads to nanostructured grafting that follows the pattern of precursor self-assembly, while the direct grafting in organic solvent (without the self-assembly step) results in random grafting of the surface [116]. a, b were adapted from [108] with permission from The Royal Society of Chemistry. c adapted with permission from [116]; copyright (C) 2016 American Chemical Society

Nanoporous (35-200 nm) patterns (Fig. 22b) were also reported when combining the grafting and electrochemical etching of HOPG surface [117]. Performing +0.8 to $-0.8 \mathrm{~V} \mathrm{CV}$ cycles at $20 \mathrm{mV} / \mathrm{s}$ scan rate in $0.5,2$ or $5 \mathrm{M} \mathrm{HCl}$ solutions containing 4-carboxyphenyldiazonium salt, Cui et al. observed gradual increase of the dimension of the etched pores. 
Grafted adlayers can be used to tune on-surface self-assembly. Electrodeposition of gold nanostructures on pristine HOPG substrate primarily occur at step-edges [78] while $\mathrm{NH}_{2}$-functionalized grafted adlayer promotes uniform deposition of $\mathrm{Au}$ nanoparticles on top of it [118].

Examples of molecular self-assemblies on arylated HOPG are also known. For such studies the ability of STM tip to degraft covalently attached aryls selectively, under very mild conditions and with nanoscale precision opened new exciting possibilities that are only beginning to be explored. Thus, local removal of grafted species allows to direct and study ripening of $2 \mathrm{D}$ crystals that were kinetically trapped in the framework of a low-density grafted adlayer (Fig. 23a, b, [109]). Manually degrafted corrals can be used to localize supramolecular assemblies (Fig. 23c-e, g, [110, 120]). And finally, such corrals can be used as nanoreactors with controlled shape, size and chemical functionality. For example, we have already demonstrated the use of such corrals for self-assembly of in situ generated EC-active species under full electrochemical control [81], and for the investigation of tip-induced and photochemical polymerization of diacetylenes under nanoconfinement (Fig. 23f, h, [119]).

\section{Summary and Conclusions}

In this contribution, we have summarized a variety of molecule-based surface chemistry approaches on graphene and graphite. Several of the chemistries rely on the formation of well-ordered monolayers that undergo a postfunctionalization, be it orthogonal to the surface or in the monolayer plane. Nowadays, the formation of two-dimensional polymers is one of the most popular applications of on-surface chemistry, driven by the prospect of new and interesting properties that may arise for these new materials. Several types of protocols can induce on-surface reactions

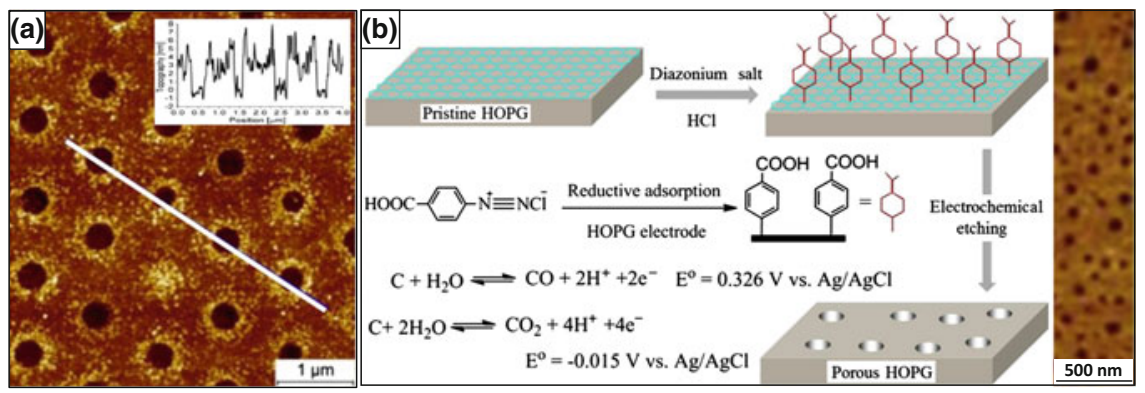

Fig. 22 Nanopatterns formed via: a electrochemical reduction of a diazonium salt on ITO electrode covered with polystyrene beads [112]; b simultaneous aryl grafting and graphite oxidation [117]. a adapted with permission from [112]; copyright (C) 2016 American Chemical Society. b adapted from [117], copyright (C) 2016, with permission from Elsevier 

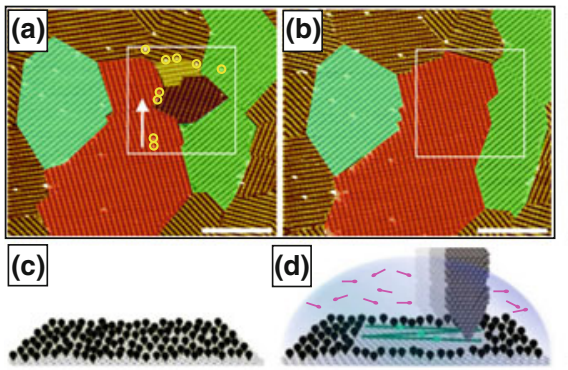

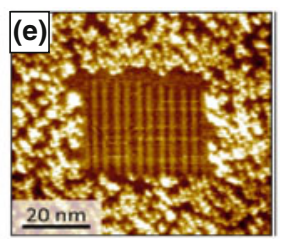

$(\mathrm{g})$

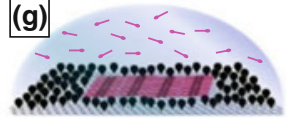

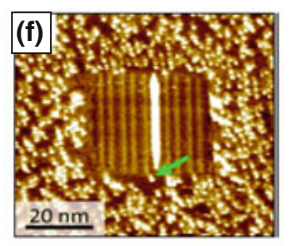

(h)

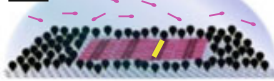

Fig. 23 The power of controlled degrafting: a application of manual removal of grafts (marked with yellow circles) to promote b local (marked with the white rectangle) Ostwald ripening of physisorbed supramolecular assembly [109]. In situ STM lithography d of a dense adlayer of covalent grafts $\mathbf{c}$ for directed self-assembly $(\mathbf{e}, \mathbf{g})$ and tip induced polymerization $(\mathbf{f}, \mathbf{h})$ of 10,12-pentacosadiynoic acid [119]. a, b were adapted from [109] with permission from The Royal Society of Chemistry. c, d, $\mathbf{g}, \mathbf{h}$ adapted with permission from [120]; copyright (C) 2016 American Chemical Society. e, f were adapted from [119] with permission, copyright (C) 2017, from The Royal Society of Chemistry

such as thermal, light, or electrochemical activation. One aspect that has not received as much attention yet is the use of surfaces to affect the product distribution of a chemical reaction that occurs in solution. Initial reports suggest that such combination of on-surface self-assembly and combinatorial chemistry might be complementary to existing synthetic strategies. Also, the complexity and, hence, the potential of covalent functionalization of carbon surfaces is not yet fully realized. Although such chemistries are known for more than two decades, their investigation using scanning probe microscopy techniques and the nanoscale characterization will lead to new patterning strategies. Combining different on-surface reactivity protocols may lead to a new generation of smart surfaces and materials.

Acknowledgements This work is supported by the Fund of Scientific Research-Flanders (FWO), KU Leuven-Internal Funds, Belgian Federal Science Policy Office (IAP-7/05), European Research Council under the European Union's Seventh Framework Program (FP7/2007-2013)/ ERC Grant Agreement No. 340324, and from the European Union Framework Program for Research and Innovation Horizon 2020 as a Future and Emerging Technologies Action (FET Open) under Grant Agreement No. 664878 (2D-INK).

\section{References}

1. Hughes, G., Westmacott, K., Honeychurch, K., Crew, A., Pemberton, R., Hart, J.: Biosensors 6, 50 (2016)

2. Shivananju, B.N., Yu, W., Liu, Y., Zhang, Y., Lin, B., Li, S., Bao, Q.: Adv. Funct. Mater. n/ a (2016)

3. Ferrari, A.C., Bonaccorso, F., Fal'ko, V., Novoselov, K.S., Roche, S., Boggild, P., Borini, S., Koppens, F.H.L., Palermo, V., Pugno, N., Garrido, J.A., Sordan, R., Bianco, A., Ballerini, L., Prato, M., Lidorikis, E., Kivioja, J., Marinelli, C., Ryhanen, T., Morpurgo, A., 
Coleman, J.N., Nicolosi, V., Colombo, L., Fert, A., Garcia-Hernandez, M., Bachtold, A., Schneider, G.F., Guinea, F., Dekker, C., Barbone, M., Sun, Z., Galiotis, C., Grigorenko, A. N., Konstantatos, G., Kis, A., Katsnelson, M., Vandersypen, L., Loiseau, A., Morandi, V., Neumaier, D., Treossi, E., Pellegrini, V., Polini, M., Tredicucci, A., Williams, G.M., Hee Hong, B., Ahn, J.-H., Min Kim, J., Zirath, H., van Wees, B.J., van der Zant, H., Occhipinti, L., Di Matteo, A., Kinloch, I.A., Seyller, T., Quesnel, E., Feng, X., Teo, K., Rupesinghe, N., Hakonen, P., Neil, S.R.T., Tannock, Q., Lofwander, T., Kinaret, J.: Nanoscale 7, 4598 (2015)

4. Huang, H., Wang, X.: Journal of Materials Chemistry A 2, 6266 (2014)

5. Hu, F.-Y., Zhang, X.-M., Wang, X.-C., Wang, S., Wang, H.-Q., Duan, W.-B., Zeng, Q.-D., Wang, C.: ACS Appl. Mater. Interfaces. 5, 1583 (2013)

6. Wei, X., Tong, W., Fidler, V., Zimmt, M.B.: J. Colloid Interface Sci. 387, 221 (2012)

7. Du, P., Jaouen, M., Bocheux, A., Bourgogne, C., Han, Z., Bouchiat, V., Kreher, D., Mathevet, F., Fiorini-Debuisschert, C., Charra, F., Attias, A.-J.: Angew. Chem. Int. Ed. 53, 10060 (2014)

8. Bléger, D., Mathevet, F., Kreher, D., Attias, A.-J., Bocheux, A., Latil, S., Douillard, L., Fiorini-Debuisschert, C., Charra, F.: Angew. Chem. Int. Ed. 50, 6562 (2011)

9. Du, P., Bléger, D., Charra, F., Bouchiat, V., Kreher, D., Mathevet, F., Attias, A.-J.: Beilstein J. Nanotechnol. 6, 632 (2015)

10. Le Liepvre, S., Du, P., Kreher, D., Mathevet, F., Attias, A.-J., Fiorini-Debuisschert, C., Douillard, L., Charra, F.: ACS Photonics 3, 2291 (2016)

11. Spitzer, S., Rastgoo-Lahrood, A., Macknapp, K., Ritter, V., Sotier, S., Heckl, W.M., Lackinger, M.: Chem. Commun. (2017)

12. Liu, X.-H., Guan, C.-Z., Ding, S.-Y., Wang, W., Yan, H.-J., Wang, D., Wan, L.-J.: J. Am. Chem. Soc. 135, 10470 (2013)

13. Giancarlo, L.C., Flynn, G.W.: Acc. Chem. Res. 33, 491 (2000)

14. Schull, G., Ness, H., Douillard, L., Fiorini-Debuisschert, C., Charra, F., Mathevet, F., Kreher, D., Attias, A.-J.: J. Phys. Chem. C 112, 14058 (2008)

15. Fang, Y., Ghijsens, E., Ivasenko, O., Cao, H., Noguchi, A., Mali, K.S., Tahara, K., Tobe, Y., De Feyter, S.: Nat. Chem. 8, 711 (2016)

16. Plas, J., Waghray, D., Adisoejoso, J., Ivasenko, O., Dehaen, W., De Feyter, S.: Chem. Commun. 51, 16338 (2015)

17. Dienstmaier, J.F., Gigler, A.M., Goetz, A.J., Knochel, P., Bein, T., Lyapin, A., Reichlmaier, S., Heckl, W.M., Lackinger, M.: ACS Nano 5, 9737 (2011)

18. Hu, Y., Goodeal, N., Chen, Y., Ganose, A.M., Palgrave, R.G., Bronstein, H., Blunt, M.O.: Chem. Commun. 52, 9941 (2016)

19. He, J., Fang, C., Shelp, R.A., Zimmt, M.B.: Langmuir 33, 459 (2017)

20. Li, Y., Wan, J., Deng, K., Han, X., Lei, S., Yang, Y., Zheng, Q., Zeng, Q., Wang, C.: J. Phys. Chem. C 115, 6540 (2011)

21. Liu, X.-H., Guan, C.-Z., Zheng, Q.-N., Wang, D., Wan, L.-J.: J. Chem. Phys. 142, 101905 (2015)

22. Dong, W.-L., Wang, L., Ding, H.-M., Zhao, L., Wang, D., Wang, C., Wan, L.-J.: Langmuir 31, 11755 (2015)

23. Sun, X., Fan, L., Zhou, X., Tian, W.Q., Guo, Z., Li, Z., Li, X., Lei, S.: Chem. Commun. 51, $5864(2015)$

24. Xu, L., Yu, Y., Lin, J., Zhou, X., Tian, W.Q., Nieckarz, D., Szabelski, P., Lei, S.: Nanoscale 8, 8568 (2016)

25. Yue, J.-Y., Markoulides, M., Regan, A.C., Li, S.-Y., Chronakis, N., Gourdon, A., Chen, T., Yan, H.-J., Wang, D.: Chem. Commun. 53, 428 (2017)

26. Yu, Y., Zheng, Y., Lei, S.: J. Phys. Chem. C 121, 593 (2017) 
27. Gong, Z., Yang, B., Lin, H., Tang, Y., Tang, Z., Zhang, J., Zhang, H., Li, Y., Xie, Y., Li, Q., Chi, L.: ACS Nano 10, 4228 (2016)

28. Yu, Y., Yang, L., Liu, C., Tian, W.Q., Wang, Y., Lei, S.: Chem. Commun. 52, 8317 (2016)

29. Mo, Y.-P., Liu, X.-H., Sun, B., Yan, H.-J., Wang, D., Wan, L.-J.: Phys. Chem. Chem. Phys. 19, 539 (2017)

30. Xu, L., Cao, L., Guo, Z., Zha, Z., Lei, S.: Chem. Commun. 51, 8664 (2015)

31. Ciesielski, A., El Garah, M., Haar, S., Kovaříček, P., Lehn, J.-M., Samorì, P.: Nat Chem 6, 1017 (2014)

32. Yu, Y., Lin, J., Lei, S.: RSC Adv. 7, 11496 (2017)

33. Yu, Y., Lin, J., Wang, Y., Zeng, Q., Lei, S.: Chem. Commun. 52, 6609 (2016)

34. Higuchi, R., Tanoue, R., Sakaguchi, K., Yanai, K., Uemura, S., Kunitake, M.: Polymer 54, $3452(2013)$

35. de la Peña Ruigómez, A., Rodríguez-San-Miguel, D., Stylianou, K.C., Cavallini, M., Gentili, D., Liscio, F., Milita, S., Roscioni, O.M., Ruiz-González, M.L., Carbonell, C., Maspoch, D., Mas-Ballesté, R., Segura, J.L., Zamora, F.: Chem.-A Eur. J. 21, 10666 (2015)

36. Dong, W.-L., Li, S.-Y., Yue, J.-Y., Wang, C., Wang, D., Wan, L.-J.: Phys. Chem. Chem. Phys. 18, 17356 (2016)

37. Higuchi, R., Tanoue, R., Enoki, N., Miyasato, Y., Sakaguchi, K., Uemura, S., Kimizuka, N., Kunitake, M.: Chem. Commun. 48, 3103 (2012)

38. Li, H., Xu, X., Shang, J., Li, J., Hu, X., Teo, B.K., Wu, K.: J. Phys. Chem. C 116, 21753 (2012)

39. Li, Y., Liu, C., Xie, Y., Li, X., Fan, X., Yuan, L., Zeng, Q.: Chem. Commun. 49, 9021 (2013)

40. Ciesielski, A., Lena, S., Masiero, S., Spada, G.P., Samorì, P.: Angew. Chem. Int. Ed. 49, 1963 (2010)

41. Luo, D., Zhang, X., Shen, Y., Xu, J., Shu, L., Zeng, Q., Wang, C.: Chem. Commun. 50, 9369 (2014)

42. Geng, Y., Liu, M., Xue, J., Xu, P., Wang, Y., Shu, L., Zeng, Q., Wang, C.: Chem. Commun. 51, 6820 (2015)

43. Gong, Y., Zhang, S., Geng, Y., Niu, C., Yin, S., Zeng, Q., Li, M.: Langmuir 31, 11525 (2015)

44. Garah, M.E., Ciesielski, A., Marets, N., Bulach, V., Hosseini, M.W., Samori, P.: Chem. Commun. 50, 12250 (2014)

45. Kikkawa, Y., Koyama, E., Aoyagi, M., Schneider, N., Takahashi, M., Fujiwara, K., Kanesato, M.: Supramol. Chem. 23, 9 (2011)

46. Omori, K., Kikkawa, Y., Tokuhisa, H., Kanesato, M., Hiratani, K.: Colloids Surf., A 356, 58 (2010)

47. Omori, K., Kikkawa, Y., Kanesato, M., Hiratani, K.: Chem. Commun. 46, 8008 (2010)

48. Yoshihiro, K., Kazuhiro, O., Masatoshi, K., Kazuhisa, H.: Chem. Lett. 41, 1196 (2012)

49. Geng, Y., Dai, H., Chang, S., Hu, F., Zeng, Q., Wang, C.: ACS Appl. Mater. Interfaces. 7, 4659 (2015)

50. Dienstmaier, J.F., Medina, D.D., Dogru, M., Knochel, P., Bein, T., Heckl, W.M., Lackinger, M.: ACS Nano 6, 7234 (2012)

51. Yu, L., Li, Z.-B., Wang, D.: Chem. Commun. 52, 13771 (2016)

52. Yue, J.-Y., Mo, Y.-P., Li, S.-Y., Dong, W.-L., Chen, T., Wang, D.: Chem. Sci. 8, 2169 (2017)

53. Ono, K., Johmoto, K., Yasuda, N., Uekusa, H., Fujii, S., Kiguchi, M., Iwasawa, N.: J. Am. Chem. Soc. 137, 7015 (2015)

54. Colson, J.W., Woll, A.R., Mukherjee, A., Levendorf, M.P., Spitler, E.L., Shields, V.B., Spencer, M.G., Park, J., Dichtel, W.R.: Science 332, 228 (2011) 
55. Liu, C., Yu, Y., Zhang, W., Zeng, Q., Lei, S.: Chem.-A Eur. J. 22, 18412 (2016)

56. Miura, A., De Feyter, S., Abdel-Mottaleb, M.M.S., Gesquière, A., Grim, P.C.M., Moessner, G., Sieffert, M., Klapper, M., Müllen, K., De Schryver, F.C.: Langmuir 19, 6474 (2003)

57. Grim, P.C.M., Vanoppen, P., Rücker, M., Feyter, S.D., Valiyaveettil, S., Moessner, G., Müllen, K., Schryver, F.C.D.: J. Vac. Sci. Technol. B: Microelectron. Nanometer Struct. Process. Meas. Phenom. 15, 1419 (1997)

58. Bléger, D., Ciesielski, A., Samorì, P., Hecht, S.: Chem.-A Eur. J. 16, 14256 (2010)

59. Jacob, H., Ulrich, S., Jung, U., Lemke, S., Rusch, T., Schutt, C., Petersen, F., Strunskus, T., Magnussen, O., Herges, R., Tuczek, F.: Phys. Chem. Chem. Phys. 16, 22643 (2014)

60. Tahara, K., Inukai, K., Adisoejoso, J., Yamaga, H., Balandina, T., Blunt, M.O., De Feyter, S., Tobe, Y.: Angew. Chem. Int. Ed. 52, 8373 (2013)

61. Shen, Y.-T., Guan, L., Zhu, X.-Y., Zeng, Q.-D., Wang, C.: J. Am. Chem. Soc. 131, 6174 (2009)

62. Yamauchi, M., Kanao, N., Adhikari, B., Karatsu, T., Yagai, S.: Chem. Lett. 46, 111 (2017)

63. Tahara, K., Nakatani, K., Iritani, K., De Feyter, S., Tobe, Y.: ACS Nano 10, 2113 (2016)

64. Shen, Y.-T., Deng, K., Zhang, X.-M., Feng, W., Zeng, Q.-D., Wang, C., Gong, J.R.: Nano Lett. 11, 3245 (2011)

65. Liao, L.-Y., Li, Y.-B., Zhang, X.-M., Geng, Y.-F., Zhang, J.-Y., Xie, J.-L., Zeng, Q.-D., Wang, C.: J. Phys. Chem. C 118, 15963 (2014)

66. Abdel-Mottaleb, M.M.S., De Feyter, S., Gesquière, A., Sieffert, M., Klapper, M., Müllen, K., De Schryver, F.C.: Nano Lett. 1, 353 (2001)

67. Xue, J., Xu, J., Hu, F., Liao, L., Li, M., Duan, W., Zeng, Q., Wang, C.: Phys. Chem. Chem. Phys. 16, 25765 (2014)

68. Kikkawa, Y., Kihara, H., Takahashi, M., Kanesato, M., Balaban, T.S., Lehn, J.-M.: J. Phys. Chem. B 114, 16718 (2010)

69. Yokoyama, S., Hirose, T., Matsuda, K.: Langmuir 31, 6404 (2015)

70. Maeda, N., Hirose, T., Yokoyama, S., Matsuda, K.: J. Phys. Chem. C 120, 9317 (2016)

71. Bonacchi, S., El Garah, M., Ciesielski, A., Herder, M., Conti, S., Cecchini, M., Hecht, S., Samorì, P.: Angew. Chem. 127, 4947 (2015)

72. Colazzo, L., Sedona, F., Moretto, A., Casarin, M., Sambi, M.: J. Am. Chem. Soc. 138, 10151 (2016)

73. Takami, T., Ozaki, H., Kasuga, M., Tsuchiya, T., Ogawa, A., Mazaki, Y., Fukushi, D., Uda, M., Aono, M.: Angew. Chem., Int. Ed. Engl. 36, 2755 (1997)

74. Xue, Y., Zimmt, M.B.: J. Am. Chem. Soc. 134, 4513 (2012)

75. Yang, Y., Zimmt, M.B.: Langmuir 31, 12408 (2015)

76. Colson, J.W., Dichtel, W.R.: Nat. Chem. 5, 453 (2013)

77. Zhang, X.-M., Xu, S.-D., Li, M., Shen, Y.-T., Wei, Z.-Q., Wang, S., Zeng, Q.-D., Wang, C.: J. Phys. Chemi. C 116, 8950 (2012)

78. Gómez, J.J.A., Zubieta, C., Ferullo, R.M., García, S.G.: Appl. Surf. Sci. 363, 356 (2016)

79. Tang, Z., Liu, S., Wang, Z., Dong, S., Wang, E.: Electrochem. Commun. 2, 32 (2000)

80. El Garah, M., Santana Bonilla, A., Ciesielski, A., Gualandi, A., Mengozzi, L., Fiorani, A., Iurlo, M., Marcaccio, M., Gutierrez, R., Rapino, S., Calvaresi, M., Zerbetto, F., Cuniberti, G., Cozzi, P.G., Paolucci, F., Samori, P.: Nanoscale 8, 13678 (2016)

81. Huynh, T.M.T., Phan, T.H., Ivasenko, O., Mertens, S.F.L., De Feyter, S.: Nanoscale 9, 362 (2017)

82. Seo, S., Lee, K., Min, M., Cho, Y., Kim, M., Lee, H.: Nanoscale 9, 3969 (2017)

83. Griessl, S.J.H., Lackinger, M., Jamitzky, F., Markert, T., Hietschold, M., Heckl, W.M.: J. Phys. Chem. B 108, 11556 (2004)

84. Lee, S.-L., Hsu, Y.-J., Wu, H.-J., Lin, H.-A., Hsu, H.-F., Chen, C.-H.: Chem. Commun. 48, $11748(2012)$ 
85. Ivasenko, O., MacLeod, J.M., Chernichenko, K.Y., Balenkova, E.S., Shpanchenko, R.V., Nenajdenko, V.G., Rosei, F., Perepichka, D.F.: Chem. Commun. 10, 1192 (2009)

86. den Boer, D., Li, M., Habets, T., Iavicoli, P., Rowan, A.E., Nolte, R.J.M., Speller, S., Amabilino, D.B., De Feyter, S., Elemans, J.A.A.W.: Nat Chem 5, 621 (2013)

87. Masato, N., Masakazu, A., Tomonobu, N.: Jpn. J. Appl. Phys. 55(11), 4 (2016)

88. Okawa, Y., Akai-Kasaya, M., Kuwahara, Y., Mandal, S.K., Aono, M.: Nanoscale 4, 3013 (2012)

89. Okawa, Y., Mandal, S.K., Hu, C., Tateyama, Y., Goedecker, S., Tsukamoto, S., Hasegawa, T., Gimzewski, J.K., Aono, M.: J. Am. Chem. Soc. 133, 8227 (2011)

90. Nakaya, M., Okawa, Y., Joachim, C., Aono, M., Nakayama, T.: ACS Nano 8, 12259 (2014)

91. Plas, J., Ivasenko, O., Martsinovich, N., Lackinger, M., De Feyter, S.: Chem. Commun. 52, 68 (2016)

92. Stevens, F., Kolodny, L.A., Beebe, T.P.: J. Phys. Chem. B 102, 10799 (1998)

93. Fredriksson, H., Chakarov, D., Kasemo, B.: Carbon 47, 1335 (2009)

94. Artur, B., Moritz, H., Ninette, S., Stefan, S.J., Sharali, M., Fabián, P.-W., Patrice, B., Dagmar, G., Manfred, M.K.: Nanotechnology 17, 5889 (2006)

95. Stevens, F., Buehner, D., Beebe, T.P.: J. Phys. Chem. B 101, 6491 (1997)

96. Sullivan, S.P., Schnieders, A., Mbugua, S.K., Beebe, T.P.: Langmuir 21, 1322 (2005)

97. Cong, C.X., Yu, T., Ni, Z.H., Liu, L., Shen, Z.X., Huang, W.: J. Phys. Chem. C 113, 6529 (2009)

98. Xu, W., Seo, H.-K., Min, S.-Y., Cho, H., Lim, T.-S., Oh, C.-Y., Lee, Y., Lee, T.-W.: Adv. Mater. 26, 3459 (2014)

99. Son, J.G., Son, M., Moon, K.-J., Lee, B.H., Myoung, J.-M., Strano, M.S., Ham, M.-H., Ross, C.A.: Adv. Mater. 25, 4723 (2013)

100. Jin, Z., Sun, W., Ke, Y., Shih, C.-J., Paulus, G.L.C., Hua Wang, Q., Mu, B., Yin, P., Strano, M.S.: Nat. Commun. 4, 1663 (2013)

101. Zheng, Y., Wang, H., Hou, S., Xia, D.: Adv. Mater. Technol. 2, 1600237 (2017)

102. Rubio-Verdu, C., Saenz-Arce, G., Martinez-Asencio, J., Milan, D.C., Moaied, M., Palacios, J.J., Caturla, M.J., Untiedt, C.: Phys. Chem. Chem. Phys. 19, 8061 (2017)

103. Dobrik, G., Tapasztó, L., Nemes-Incze, P., Lambin, P., Biró, L.P.: Phys. Status Solidi B 247, 896 (2010)

104. Tapaszto, L., Dobrik, G., Lambin, P., Biro, L.P.: Nat Nano 3, 397 (2008)

105. Park, J., Yan, M.: Acc. Chem. Res. 46, 181 (2013)

106. Downard, A.J.: Electroanalysis 12, 1085 (2000)

107. Barrière, F., Downard, A.J.: J. Solid State Electrochem. 12, 1231 (2008)

108. Li, Z., Van Gorp, H., Walke, P., Phan, T.H., Fujita, Y., Greenwood, J., Ivasenko, O., Tahara, K., Tobe, Y., Uji-i, H., Mertens, S.F.L., De Feyter, S.: Nanoscale 9, 5188 (2017)

109. Braganca, A.M., Greenwood, J., Ivasenko, O., Phan, T.H., Mullen, K., De Feyter, S.: Chem. Sci. 7, 7028 (2016)

110. Greenwood, J., Phan, T.H., Fujita, Y., Li, Z., Ivasenko, O., Vanderlinden, W., Van Gorp, H., Frederickx, W., Lu, G., Tahara, K., Tobe, Y., Uji-i, H., Mertens, S.F.L., De Feyter, S.: ACS Nano 9, 5520 (2015)

111. Seber, G., Rudnev, A.V., Droghetti, A., Rungger, I., Veciana, J., Mas-Torrent, M., Rovira, C., Crivillers, N.: Chem.-A Eur. J. 23, 1415 (2017)

112. Nguyen, V.Q., Sun, X., Lafolet, F., Audibert, J.-F., Miomandre, F., Lemercier, G., Loiseau, F., Lacroix, J.-C.: J. Am. Chem. Soc. 138, 9381 (2016)

113. Huder, L., Rinfray, C., Rouchon, D., Benayad, A., Baraket, M., Izzet, G., Lipp-Bregolin, F., Lapertot, G., Dubois, L., Proust, A., Jansen, L., Duclairoir, F.: Langmuir 32, 4774 (2016)

114. Cao, C., Zhang, Y., Jiang, C., Qi, M., Liu, G.: ACS Appl. Mater. Interfaces. 9, 5031 (2017)

115. Ejigu, A., Kinloch, I.A., Dryfe, R.A.W.: ACS Appl. Mater. Interfaces. 9, 710 (2017) 
116. Xia, Z., Leonardi, F., Gobbi, M., Liu, Y., Bellani, V., Liscio, A., Kovtun, A., Li, R., Feng, X., Orgiu, E., Samorì, P., Treossi, E., Palermo, V.: ACS Nano 10, 7125 (2016)

117. Cui, L., Xu, Y., Liu, B., Yang, W., Song, Z., Liu, J.: Carbon 102, 419 (2016)

118. Gonzalez, M.C.R., Orive, A.G., Salvarezza, R.C., Creus, A.H.: Phys. Chem. Chem. Phys. 18, 1953 (2016)

119. Verstraete, L., Hirsch, B.E., Greenwood, J., De Feyter, S.: Chem. Commun. 53, 4207 (2017)

120. Verstraete, L., Greenwood, J., Hirsch, B.E., De Feyter, S.: ACS Nano 10, 10706 (2016)

Open Access This chapter is licensed under the terms of the Creative Commons Attribution 4.0 International License (http://creativecommons.org/licenses/by/4.0/), which permits use, sharing, adaptation, distribution and reproduction in any medium or format, as long as you give appropriate credit to the original author(s) and the source, provide a link to the Creative Commons license and indicate if changes were made.

The images or other third party material in this chapter are included in the chapter's Creative Commons license, unless indicated otherwise in a credit line to the material. If material is not included in the chapter's Creative Commons license and your intended use is not permitted by statutory regulation or exceeds the permitted use, you will need to obtain permission directly from the copyright holder.

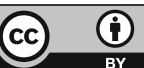

